\title{
Phase and Amplitude Calibration of Rotating Equispaced Circular Array for Geostationary Microwave Interferometric Radiometers- Simulation Results and Discussion
}

\author{
Xi Guo, Student Member, IEEE, Adriano Camps, Fellow, IEEE, Hyuk Park, Senior Member, IEEE \\ Hao Liu, Member, IEEE, Cheng Zhang, Member, IEEE, Ji Wu, Fellow, IEEE
}

\begin{abstract}
In the theoretical part of this work, a calibration strategy for both phase and amplitude calibration of rotating equispaced circular array based on redundant space calibration was introduced. It avoids the challenging implementation of conventional noise-injection network, especially for a large-scale array, by using the strong self-consistency accomplished by array rotation. This study presents comprehensive simulation results of the proposed calibration strategy, where calibration residuals and image reconstruction errors are evaluated. The phase calibration shows outstanding performance, while the amplitude calibration is found to be biased due to the logarithms used to linearize the system of calibration equations, and the resulting image reconstruction bias is scene-dependent. A bias correction method is proposed accordingly, which requires an a priori regional brightness temperature within the observation scene. The performance of both phase and amplitude calibration take advantage of increasing the number of elements, which promises the system scalability for high spatial resolution applications.
\end{abstract}

Index Terms-Microwave radiometry, amplitude calibration, phase calibration, redundant baselines, circular array, rotational sampling, synthetic aperture interferometric radiometer.

\section{INTRODUCTION}

$\mathbf{I}$ $\mathrm{N}$ the theoretical part of this two-part paper [1], a novel calibration strategy for both phase and amplitude calibration based on redundant space calibration was proposed. By using the unique structure of equispaced circular array and rotational sampling strategy, calibration can be conducted

$\mathrm{Xi}$ Guo is with the School of Electronic, Electrical and Communication Engineering, University of Chinese Academy of Sciences, Beijing 100049, China, and also with the Key Laboratory of Microwave Remote Sensing, National Space Science Center, Chinese Academy of Sciences, Beijing 100190, China (e-mail: xi_guo@hotmail.com).

Adriano Camps and Hyuk Park are with the Department of Signal Theory and Communications, Universitat Politècnica de Catalunya, Unidad de Excellencia María de Maeztu-CommSensLab, and Universitat Politècnica de Catalunya - BarcelonaTech, and IEEC/CTE-UPC, 08034 Barcelona, Spain (e-mail: camps@tsc.upc.edu; park.hyuk@upc.edu)

Hao Liu, Cheng Zhang, and Ji Wu are with the Key Laboratory of Microwave Remote Sensing, National Space Science Center, Chinese Academy of Sciences, Beijing 100190, China (e-mail: liuhao@mirslab.cn; zhangcheng@mirslab.cn; wuji@nssc.ac.cn).

This work was supported for NSSC's Contribution in part by the National Natural Science Foundation of China under Grant 41675035, in part by the Special Fund for Chinese Meteorological Research in the Public Interest under Grant GYHY201506023, and in part by the Beijing Science Project under Grant Z161100002616033. The UPC's Contribution was supported by the Spanish Ministry of Science, Innovation and Universities, "SPOT: Sensing with Pioneering Opportunistic Techniques," under Grant RTI2018-099008-BC21/AEI/10.13039/501100011033. (Corresponding author: Hao Liu.) independently, without the help of a noise-injection network, which is the main benefit of this calibration strategy. Besides, the irregular phase ambiguity that inherently exists in the retrieved phase is degenerated to the fixed $\pi$-ambiguity, so that sophisticated phase unwrapping technique is not needed. As the conventional noise-injection calibration method for interferometric synthetic aperture radiometers is challenging to be implemented on a large-scale array, the proposed calibration strategy provides an alternative calibration solution for geostationary microwave interferometric radiometers.

This paper is devoted to evaluating the proposed calibration strategy's performance, and it is organized as follows.

In Section II, the simulation model and its configurations are described including: A) the brightness temperature and the visibility function; B) the separable and non-separable factors; C) the additive noise term; and D) the image reconstruction.

In Section III, the noise analysis and the design of weighting functions are discussed: A) since the noise term is directly added to the complex correlation, its impact on both the phase and the amplitude of the measured complex correlation is evaluated by means of Monte Carlo simulations, and B) the weighting functions for both phase and amplitude calibration are designed based on the noise behavior.

In Section IV, the simulation results of the phase calibration are presented and discussed. A) The initial guess's effectiveness is first examined, which provides important insights into the self-consistency achieved by the proposed calibration strategy. B) Then, the phase calibration performance is evaluated when the averaging method is not considered, and the properties of the retrieved phases are discussed. C) Finally, the phase calibration performance with averaging is presented and discussed in terms of: 1) phase calibration residuals, where different kinds of averaging methods are compared and evaluated, and 2) image reconstruction errors, where the effects of residual separable and non-separable phase errors are discussed.

In Section $\mathrm{V}$, the simulation results of the amplitude calibration are presented and discussed. A) The amplitude calibration performance is evaluated when averaging method is not considered, and the amplitude calibration bias and its corresponding image reconstruction bias is found. B) The amplitude calibration performance with averaging is presented and discussed in the following aspects: 1) amplitude calibra- 
tion residuals, which are evaluated in terms of calibration bias and uncertainty, different kinds of averaging methods are also compared and evaluated; 2) image reconstruction errors, where the amplitude calibration bias is recognized as the main image reconstruction error source.

In Section VI, a study on the amplitude calibration bias and its correction is presented. A) First, the origin of the image reconstruction bias is discussed, where the amplitude calibration bias is evaluated from the perspective of same-length baseline groups, and a scene-dependent image reconstruction error is found by building a constantly biased visibility model. B) Then, the relationship between the amplitude calibration bias and its corresponding image reconstruction bias is validated. C) Finally, a bias correction method is proposed and validated.

Lastly, important discussions and conclusions are summarized in Section VII and Section VIII, respectively.

\section{Simulation CONFIGURATiOns}

Following the definition made in Section II-A of the theoretical part of this paper [1], the complex cross-correlation of two receiving channels can be extended to include the noise contributions, and the non-separable complex gain factor is also considered:

$$
\begin{aligned}
V_{k l}^{\mathrm{raw}} & =\gamma_{k l} \cdot \gamma_{k} \cdot \gamma_{l}^{*} \cdot V_{k l}^{\mathrm{id}}+n_{k l}^{c} \\
& \triangleq G_{k l} \cdot V_{k l}^{\mathrm{id}}+n_{k l}^{c}
\end{aligned}
$$

where $G_{k l}$ is the total complex gain factor of the baseline, $\gamma_{k, l}$ are the separable complex gain factors of the $k^{t h}$ and $l^{\text {th }}$ receiving channels, $\gamma_{k l}$ is the non-separable complex gain factor, and $n_{k l}^{c}$ is the additive zero-mean Gaussian noise at the complex correlation level.

The configurations of the basic simulation model presented in (1) are described as follows.

\section{A. Brightness Temperature and Visibility Function}

In order to reduce as much as possible the potential numerical error during the simulation, a general observation scene is considered with the following assumptions:

1) The observation scene is a flat Earth disk with the brightness temperature of $T_{0}=300 \mathrm{~K}$, which has a radius of 0.15 in the $\xi-\eta$ domain (same as the Earth disk range as observed from a geostationary orbit), and the background sky temperature is neglected.

2) No antenna tapering is applied, and antenna solid angle $\Omega_{\mathrm{A}}=1$, in order to obtain a modified brightness temperature of $300 \mathrm{~K}$ (or equivalently directivity $D_{\mathrm{A}}=11 \mathrm{~dB}$. Note that a horn antenna has a directivity of $\sim 20 \mathrm{~dB}$ when its $3 \mathrm{~dB}$ beamwidth covers the Earth disk).

3) The obliquity factor $\left(1 / \sqrt{1-\xi^{2}-\eta^{2}}\right)$ is neglected, and the corresponding introduced error is less than $1.14 \%$ within the area of $\xi^{2}+\eta^{2} \leq 0.15^{2}$.

4) The effect of spatial decorrelation can be considered by using a smeared version of the modified brightness temperature, where the modified brightness temperature is averaged along the radial direction [2]. As the observation scene is set as a flat disk, the effect of spatial decorrelation only occurs at the edge of the Earth disk, which can be neglected.

5) The receiver's physical temperature term (the Corbella term) [3] in the modified brightness temperature is neglected, since the alias-free field of view (AF-FOV) for geostationary observation can be designed to be narrow and the separation of adjacent antennas is large enough (e.g., for GIMS, the shortest baseline is designed to be $3.3 \lambda @ 50.3 \mathrm{GHz}$ [4]).

The assumptions are made to fully utilize the Fourier transform relationship between the modified brightness temperature and the visibility function; therefore, the visibility function can be analytically computed by the desired array parameters:

$$
\begin{aligned}
V\left(\rho, \theta_{s}\right) & =\int_{0}^{2 \pi} \int_{0}^{1} T^{\prime}(r, \phi) \cdot e^{-j 2 \pi \rho \cdot r \cdot \cos \left(\theta_{s}-\phi\right)} r d r d \phi \\
& =\frac{45}{\rho} \cdot J_{1}\left(\frac{3 \pi}{10} \cdot \rho\right)
\end{aligned}
$$

where the visibility function is expressed in polar coordinates, $\rho$ and $\theta_{s}$ denote the radial distance and the polar angle of nonzero-baseline visibility sampling position, respectively; $T^{\prime}(r, \phi)$ is the modified brightness temperature following the same definition as [1, eq. (2)], and $J_{1}(x)$ is the first order of Bessel function of the first kind. The detailed derivation of (2) can be found in the Appendix A.

In addition, the zero-baseline visibility $V_{0}$ can be computed by:

$$
V_{0}=\iint_{\xi^{2}+\eta^{2} \leq 0.15^{2}} T^{\prime}(\xi, \eta) d \xi d \eta=21.2 \mathrm{~K}
$$

where the modified brightness temperature $T^{\prime}(\xi, \eta)$ equals to $300 \mathrm{~K}$ within the Earth disk range.

The relationship between the amplitude of the visibility function and the radial distance of visibility sampling position (also denoted by wavelength-normalized baseline length) is illustrated in Fig. 1, where the curve has a shape that similar to the sinc function, and the main energy is concentrated in the low-spatial-frequency region. Similarly, any arbitrary brightness temperature within the Earth's disk will also have a low-pass behavior.

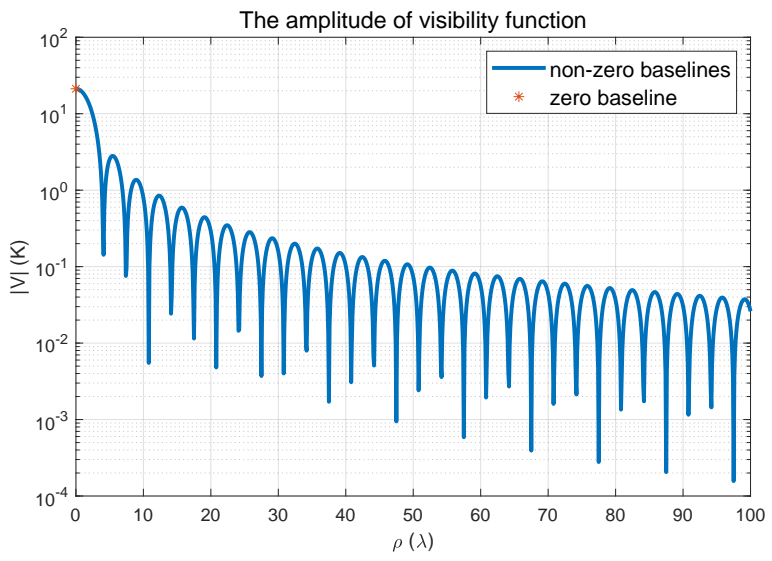

Fig. 1. Distribution of the visibility amplitude versus the wavelengthnormalized baseline length. 


\section{B. Separable and Non-separable Factors}

The total complex gain factor $G_{k l}$ can be factorized into both separable, and non-separable amplitude and phase factors, as defined in (1)

$$
\begin{aligned}
G_{k l} & =\gamma_{k l} \cdot \gamma_{k} \cdot \gamma_{l}^{*} \\
& =g_{k l} \cdot g_{k} \cdot g_{l} \cdot \exp \left[\phi_{k l}+\phi_{k}-\phi_{l}\right]
\end{aligned}
$$

where $g_{k, l}=\left|\gamma_{k, l}\right|$ and $\phi_{k, l}=\arg \left(\gamma_{k, l}\right)$, while $g_{k l}$ and $\phi_{k l}$ are the corresponding amplitude and phase terms of the nonseparable complex gain factor, respectively.

The complex correlation used in the simulations is not normalized by the zero-baseline visibility, since it is not relevant to the phase and amplitude calibration. Accordingly, the normalization factor $\sqrt{T_{A, k}}$ is avoided, and the amplitude of the separable complex gain factor is denoted by

$$
g_{k}=\frac{1}{\sqrt{T_{A, k}+T_{R, k}}}=\frac{1}{\sqrt{T_{\mathrm{sys}, k}}}
$$

where $T_{A, k}$ and $T_{R, k}$ are the antenna temperature, and the receiver's noise temperature, respectively; and their sum is denoted by the system temperature of the receiver $T_{\mathrm{sys}, k}$ [3].

During the simulations, separable phase factors are set as uniformly distributed random variables within a range from 0 to $2 \pi$. Assuming that the instrument works at the $53 \mathrm{GHz}$ frequency bands, receivers' noise temperatures are set as uniformly distributed random variables within a range from 600 to $800 \mathrm{~K}$.

Additionally, non-separable complex gain factors are mainly caused by the filters' mismatches [5] and timing errors in the correlator's samplers [6]. They are also referred to as the baseline errors, because their specific values depend on the particular pair of antennas forming the baseline. Redundant space calibration cannot retrieve the non-separable factors, so they remain as residual errors, perturbing the calibration process. Although the non-separable factors can be carefully measured during the ground testing before launch, they may still drift due to ambient temperature changes and aging. So we consider that the non-separable factors are completely unknown in our simulations.

Following the experience from the Microwave Imaging Radiometer with Aperture Synthesis (MIRAS) [7], the nonseparable factors for phase and amplitude are usually at the level of 0.15 degrees and $1 \pm 2 \%$, respectively. Thus, we assume that both of them obey Gaussian distributions: 1) $\phi_{k l} \sim N\left(0, \sigma^{2}=0.15^{2}\right)$ degrees; 2) $g_{k l} \sim N\left(1, \sigma^{2}=0.02^{2}\right)$.

\section{Additive Noise Term}

Due to the finite integration time and pre-detection noise bandwidth, the noise variance of the real and imaginary parts of the visibility measured by a baseline (antenna pairs $k^{\text {th }}$ and $l^{\text {th }}$ ) can be computed by [8], [9]

$$
\sigma_{\mathrm{R}, \mathrm{I}}^{2}=\frac{1}{2 B \tau_{e f f}}\left[\left(T_{A, k}+T_{R, k}\right)\left(T_{A, l}+T_{R, l}\right)+V_{\mathrm{R}, \mathrm{I}}^{2}-V_{\mathrm{I}, \mathrm{R}}^{2}\right]
$$

where $B$ is the receiver's pre-detection noise bandwidth, $\tau_{\text {eff }}$ is the effective integration time that depends on the signal quantization scheme used to compute the cross-correlation [10], and $V_{\mathrm{R}}$ and $V_{\mathrm{I}}$ are the real and imaginary parts of the visibility sample, respectively.

As the basic simulation model in (1) is expressed at the complex correlation level, noise should be added to the same level as well. Although the thermal noise at both visibility level and complex correlation level can be considered as zero-mean Gaussian noise, their variances are different. Note that the real and imaginary parts of the visibility samples are much smaller than the system temperature, so the last two terms in (6) can be omitted $\left(V_{\mathrm{R}, \mathrm{I}}^{2}\right.$ and $\left.V_{\mathrm{I}, \mathrm{R}}^{2}\right)$ [11]. Thus, the noise variance at the complex correlation level (normalized visibility) can be approximated by

$$
\sigma_{c}^{2} \approx \frac{1}{2 B \tau_{e f f}},
$$

and the thermal noise on both real and imagery parts of the complex correlation can be recognized by $\operatorname{Re}\left[n_{k l}^{c}\right] \sim N\left(0, \sigma_{c}^{2}\right)$ and $\operatorname{Im}\left[n_{k l}^{c}\right] \sim N\left(0, \sigma_{c}^{2}\right)$, respectively.

\section{Image Reconstruction}

The image reconstruction of the circularly sampled visibility is performed by the inverse pseudo-polar fast Fourier transform (IPPFFT) [12], [13]. The ideal zero-baseline visibility is used during the image reconstruction, since the zero-baseline visibility can be accurately calibrated by a real aperture radiometer when this calibration strategy is implemented.

In order to individually evaluate the image reconstruction error caused by the calibration residuals, radiometric error (in other words, thermal noise) and window function are not considered during the image reconstruction. The error-free reconstructed image is used as the reference image when the root-mean-square (RMS) image reconstruction error is calculated, so that the errors caused by the Gibbs ringings are partially canceled out in this way. If no specific description is given, the image reconstruction error is evaluated within the limited disk range $\xi^{2}+\eta^{2} \leq 0.13^{2}$, in order to avoid the sharp transition around the edge of the Earth disk.

Since the visibilities sampled by the equispaced circular array with an even number of elements have a redundancy factor of 2, the snap-shot visibilities are averaged by the Hermitian conjugate symmetry before the image reconstruction. Also, it should be noted that array rotation provides a great deal of measurement redundancy, but as thermal noise is not considered during the image reconstruction, averaging of redundant measurements from different snapshots is not taken into account.

\section{Noise Analysis AND Weighting Functions}

\section{A. Noise Analysis}

The calibration of complex gain factors is conducted by phase and amplitude calibration, respectively. Simplifying (1) as $V_{k l}^{\text {raw }}=V_{c o r}+n_{k l}^{c}$, the probability distributions of the resulting phase and amplitude has been given in [14] as (8) and (9) on top of next page, where $\psi_{k l}^{\text {raw }}$ and $\left|V_{k l}^{\text {raw }}\right|$ are the phase and amplitude of $V_{k l}^{\text {raw }}$, respectively; $\operatorname{erf}(\cdot)$ is the error function, and $I_{0}(\cdot)$ is the modified Bessel function of the first 


$$
\begin{gathered}
p\left(\psi_{k l}^{\mathrm{raw}}\right)=\frac{1}{2 \pi} \exp \left(-\frac{\left|V_{c o r}\right|^{2}}{2 \sigma_{c}^{2}}\right)\left\{1+\sqrt{\frac{\pi}{2}} \frac{\left|V_{c o r}\right| \cos \psi_{k l}^{\mathrm{raw}}}{\sigma_{c}} \exp \left(\frac{\left|V_{c o r}\right|^{2} \cos ^{2} \psi_{k l}^{\mathrm{raw}}}{2 \sigma_{c}^{2}}\right) \cdot\left[1+\operatorname{erf}\left(\frac{\left|V_{c o r}\right| \cos \psi_{k l}^{\mathrm{raw}}}{\sqrt{2} \sigma_{c}}\right)\right]\right\} \\
p\left(\left|V_{k l}^{\mathrm{raw}}\right|\right)=\frac{\left|V_{k l}^{\mathrm{raw}}\right|}{\sigma_{c}^{2}} \exp \left(-\frac{\left|V_{k l}^{\mathrm{raw}}\right|^{2}+\left|V_{c o r}\right|^{2}}{2 \sigma_{c}^{2}}\right) I_{0}\left(\frac{\left|V_{k l}^{\mathrm{raw}}\right|\left|V_{c o r}\right|}{\sigma_{c}^{2}}\right)
\end{gathered}
$$

kind with zero order. Note that the phase of $V_{\text {cor }}$ is set to zero for simplicity.

The curves of these two kinds of probability density functions for different signal-to-noise ratio (SNR) are shown in Fig. 2 , where the SNR of complex correlation is defined following the same convention as [14]: SNR $=\left|V_{c o r}\right| / \sigma_{c}$. The standard deviations in both cases are related to the SNR, which means that the measurements will be less corrupted under high-SNR conditions. It also can be noticed that the probability density function of the measured phase is centered at the ideal phase with a symmetric distribution, and when the signal vanishes $\left(\left|V_{\text {cor }}\right| \rightarrow 0\right)$, it degenerates into a uniform distribution. The probability density function of the measured amplitude is also referred to as a Rician distribution, and it degenerates into a Rayleigh distribution when the signal vanishes.

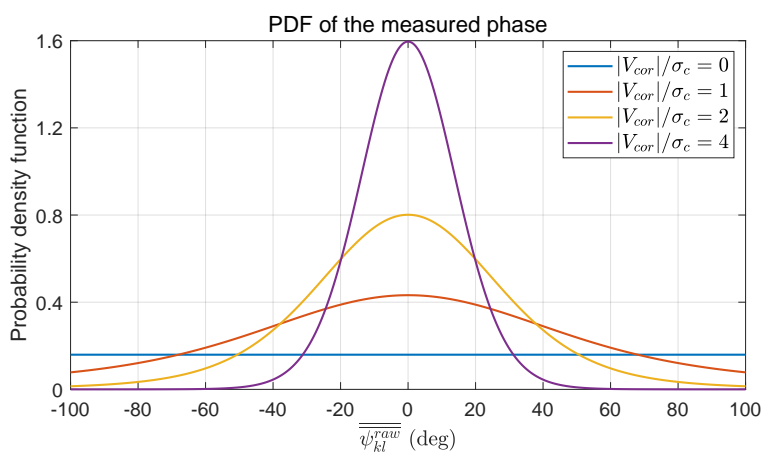

(a)

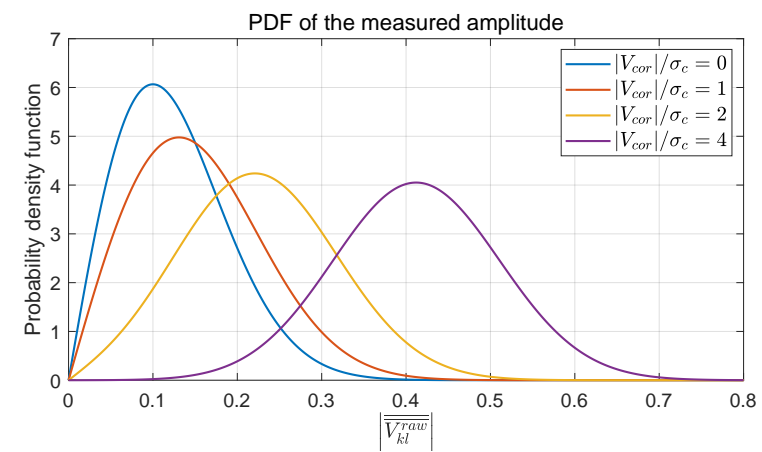

(b)

Fig. 2. Probability density function curves, (a) measured phase and (b) measured amplitude. The standard deviation of noise $\sigma_{c}$ is set to 0.1 in each case just for illustration, the noise level is far less than this value in the actual situation.

The basic calibration model under noise conditions can be rewritten by taking the phase and modulus of both sides of (1), respectively

$$
\psi_{k l}^{\mathrm{raw}}=\phi_{k l}+\phi_{k}-\phi_{l}+\psi_{k l}^{\mathrm{id}}+n_{k l}^{\phi},
$$

$$
\left|V_{k l}^{\mathrm{raw}}\right|=\left|\gamma_{k l} \cdot \gamma_{k} \cdot \gamma_{l}^{*} \cdot V_{k l}^{\mathrm{id}}+n_{k l}^{c}\right| .
$$

Since the amplitude calibration requires the unknowns to be linearly separated, (11) needs to be further linearized by taking the natural logarithm of both sides

$$
\begin{aligned}
\ln \left|V_{k l}^{\mathrm{raw}}\right| & =\ln \left|\gamma_{k l} \cdot \gamma_{k} \cdot \gamma_{l}^{*} \cdot V_{k l}^{\mathrm{id}} \cdot\left(1+\frac{n_{k l}^{c}}{\gamma_{k l} \cdot \gamma_{k} \cdot \gamma_{l}^{*} \cdot V_{k l}^{\mathrm{id}}}\right)\right| \\
& =\ln \left(g_{k l}\right)+\ln \left(g_{k}\right)+\ln \left(g_{l}\right)+\ln \left(\left|V_{k l}^{\mathrm{id}}\right|\right)+n_{k l}^{A}
\end{aligned}
$$

where $n_{k l}^{\phi}$ and $n_{k l}^{A}$ are the phase and amplitude noises resulting from the additive thermal noise at the complex correlation level. Note that, for the sake of simplicity, $n_{k l}^{A}$ is referred to as amplitude noise; as a matter of fact, it is the noise term in the natural logarithm of the amplitude of the measured complex correlation.

At this stage, the random noise terms $n_{k l}^{\phi}$ and $n_{k l}^{A}$ cannot be approximated by the Gaussian noises anymore. As they are written as additive terms in (10) and (12), their analytical expressions can be described as

$$
\begin{aligned}
n_{k l}^{\phi} & =\arg \left[\gamma_{k l} \cdot \gamma_{k} \cdot \gamma_{l}^{*} \cdot V_{k l}^{\mathrm{id}}+n_{k l}^{c}\right]-\arg \left[\gamma_{k l} \cdot \gamma_{k} \cdot \gamma_{l}^{*} \cdot V_{k l}^{\mathrm{id}}\right], \\
n_{k l}^{A} & =\ln \left|\gamma_{k l} \cdot \gamma_{k} \cdot \gamma_{l}^{*} \cdot V_{k l}^{\mathrm{id}}+n_{k l}^{c}\right|-\ln \left|\gamma_{k l} \cdot \gamma_{k} \cdot \gamma_{l}^{*} \cdot V_{k l}^{\mathrm{id}}\right| \\
& =\ln \left|1+\frac{n_{k l}^{c}}{\gamma_{k l} \cdot \gamma_{k} \cdot \gamma_{l}^{*} \cdot V_{k l}^{\mathrm{id}}}\right| .
\end{aligned}
$$

Although neither the phase noise nor the amplitude noise obeys a predefined probability distribution, their statistics are estimated by Monte Carlo simulations as shown in Fig. 3 . Since the probability density function of the measured phase has a symmetric shape about its ideal phase, the phase noise is a zero-mean random noise. The standard deviation of the phase noise has an upper limit when the measured phase degenerates into a uniform distribution $\left(V_{c o r} \rightarrow 0\right)$; thus, its upper limit can be calculated by $2 \pi / \sqrt{12}$. Besides, the mean value of the amplitude noise has a linear relationship with respect to SNR when the SNR is smaller than $\sim 0 \mathrm{~dB}$. In addition, its standard deviation has an upper limit of 0.64 . The detailed explanations of these properties can be found in the Appendix B.

\section{B. Weighting Functions}

For both phase and amplitude noises, it is obvious that the noise level is closely related to SNR of the measured complex correlation. As a general trend shown in Fig. 1, the amplitude of the ideal visibility decreases with increasing baseline length. Considering that the variance of the additive thermal noise 


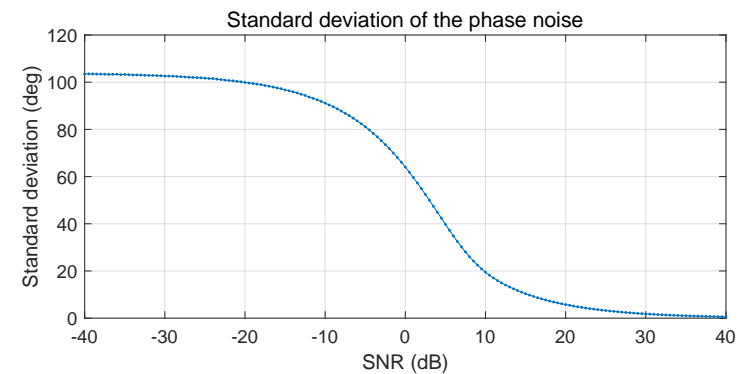

(a)

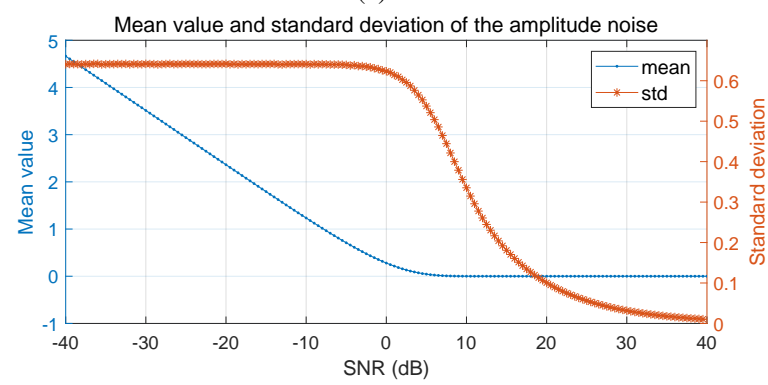

(b)

Fig. 3. Statistics of (a) phase noise and (b) amplitude noise. Note that SNR $=$ $\left|V_{\text {cor }}\right| / \sigma_{c}$, where $\sigma_{c}$ is the standard deviation of both real and imaginary part of $n_{k l}^{c}$. The vertical axis of (b) does not have any unit, since the amplitude noise is evaluated in logarithmic form.

is approximately constant for each baseline's measurement, the effective information (amplitude and phase) carried by the long baselines will unavoidably suffer from noise corruption. Besides, the baseline that samples a large-magnitude visibility will have a better SNR, and those high-SNR baselines usually have a relatively short baseline length.

Since the visibility noise is assumed to be uncorrelated between different baselines ${ }^{1}$, the noise covariance matrix becomes a diagonal matrix for both the phase and amplitude calibration, and its diagonal components can be approximated by the inverse of the variances of the measurements $\left(W_{i}=1 / \sigma_{i}^{2}\right)$ [16]. After computing the standard deviation of the phase and amplitude noise, respectively, with respect to SNR by the means of Monte Carlo simulation, it is convenient to build a look-up table or interpolant of the noise variance with SNR as the independent variable. The SNR for each baseline can be easily estimated by the system configuration and the actual measurement: 1) the noise variance can be estimated from the predefined system parameters using (7); and 2) the signal amplitude can be estimated from the averaged amplitude of the measurements of the redundant baselines.

Fig. 4 shows the normalized weighting functions calculated for the flat Earth disk observation scene. The long baselines are given smaller weight in both calibration schemes, which is achieved by the inverse variances and the SNR distribution along baselines. Note that the amplitude noise will gradually introduce a non-negligible positive bias when the baseline's SNR is less than $5 \mathrm{~dB}$, but the weights for those baselines are not larger than 0.1. Although the signal amplitudes of the redundant baselines are not exactly identical due to the

\footnotetext{
${ }^{1}$ Actually they are correlated, but the correlation can be neglected when the natural scene is being observed, see [11], [15] for details.
}

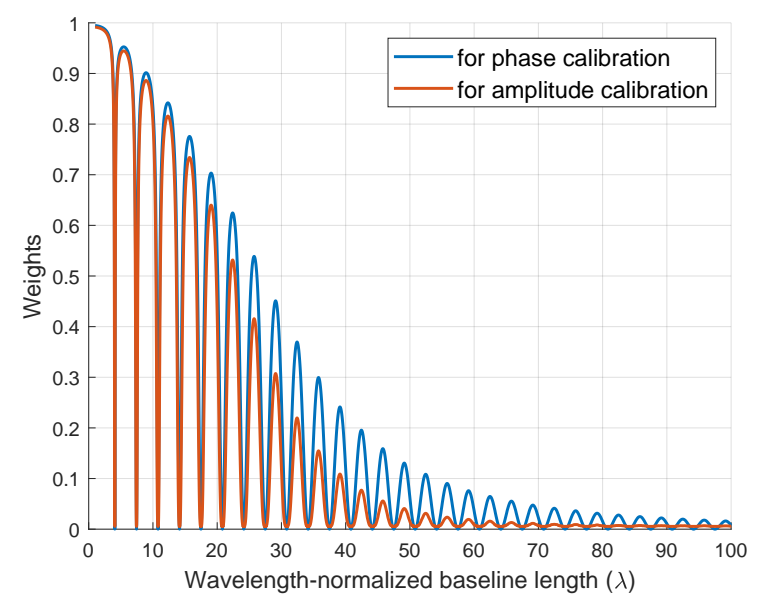

Fig. 4. Weighting functions for phase and amplitude calibration, the standard deviation of visibility noise $\sigma_{\mathrm{R}, \mathrm{I}}$ is set to $0.1 \mathrm{~K}$.

different receiver's amplitude factors, identical weights are applied to the group of same-length baselines.

It is worth mentioning that the amplitude of the measured complex correlation itself can be used as an alternate weighting for both calibration schemes, since it inherently gives smaller weights on the long baselines, and it is more selective on the high-SNR baselines than the inverse variances. Moreover, the inverse baseline length can be used as a substitute for a similar reason.

\section{Simulation Results of Phase Calibration}

Although the ideal phases of each same-length baseline group has been included in the unknowns to be solved by minimization problem, the ideal phase of every single baseline can also be estimated using the relationship presented in (10):

$$
\psi_{k l}^{\mathrm{cal}}=\psi_{k l}^{\mathrm{raw}}-\left(\hat{\phi}_{k}-\hat{\phi}_{l}\right)
$$

where $\hat{\phi}_{k, l}$ are the estimated separable phase factors, and the non-separable phase factor remains as the phase calibration residual:

$$
\epsilon_{k l}^{\psi}=\left(\phi_{k}-\phi_{l}\right)-\left(\hat{\phi}_{k}-\hat{\phi}_{l}\right)+\phi_{k l} \text {. }
$$

The ideal phase of each baseline estimated by (15) is denoted as the baseline-related phase in the following analysis, in order to be distinguished from the directly estimated ideal phase.

The phase calibration suffers from the fixed $\pi$-ambiguity, as derived in [1]. Assuming that a priori unambiguous phases have been obtained by a single image reconstruction, the ambiguity existed in the phase calibration results is removed before further analysis.

The averaging and standard deviation of the phase are calculated by the circular statistics [17] during the calibration process and analysis.

\section{A. Initial Guess}

Before the phase calibration results are presented, it is necessary to examine the effectiveness of the initial guess 
obtained by the method as derived in [1]. A typical example of the residual phase errors in the initial guess is illustrated in Fig. 5.

For the estimated separable phase factors, similar to the results presented in [18], the residual phase errors are amplified gradually along the array, but they also appear in a symmetric shape with respect to the index of antenna elements. In order to show this property more clearly, those errors from the first initial guess are displayed in the array's layout, as illustrated in Fig. 6. It can be found that the residual phase errors are strongly related to the element's separation from the reference element (Ant. 1), they grow gradually and culminate at the symmetric position of the reference element (around Ant. 76), then decrease to zero at the end of the array which is also the beginning of the array. As compared to the first initial guess, the second initial guess provides a distinctly improved estimation, because more baselines are allowed to participate in the minimization problem.

Regarding to the directly estimated ideal phases, most of the residuals are less than $10^{\circ}$, but some of them are up to $70^{\circ}$ because of the low SNR at the corresponding baselines.

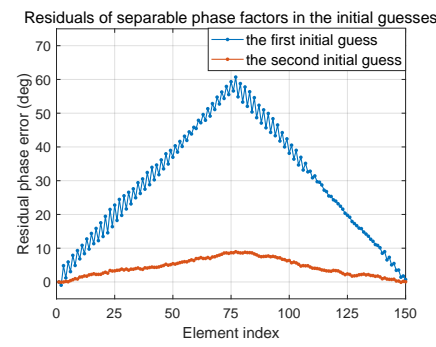

(a)

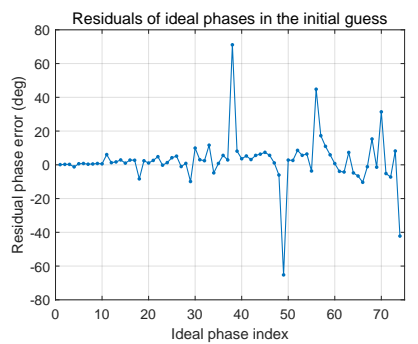

(b)
Fig. 5. Sample residual phase errors in the initial guess, (a) estimated separable phase factors, and (b) directly estimated ideal phases. The simulation is conducted by an array with 150 elements, adjacent element spacing $d_{s}=1 \lambda$. The estimated separable phase factors are normalized to the true phase of the first element, since there is one degree of freedom in the solution of separable phase factors.

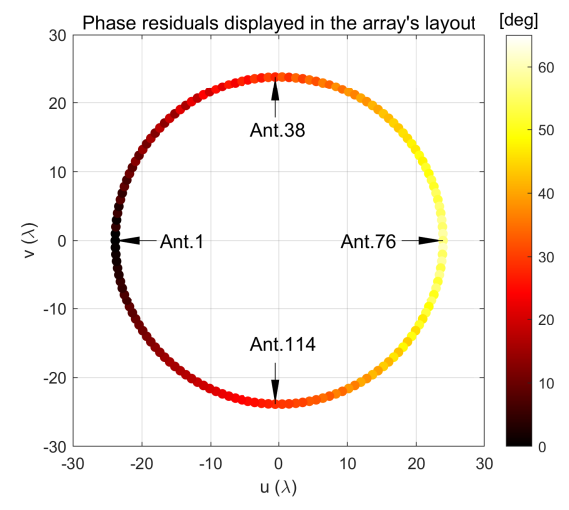

Fig. 6. Sample residuals of the estimated separable phase factors in the first initial guess. The antenna elements are indexed clockwisely.

\section{B. Single Solution}

The single solution is obtained by conducting the phase retrieval with all groups of same-length baselines involved, but the averaging of neither redundant measurements, nor solutions is carried out. A typical example of the residual phase errors in a single solution is shown in Fig. 7, where the residuals of the estimated separable phase factors are further reduced by including more measurements in the minimization problem, and the effect of error amplification is also alleviated.

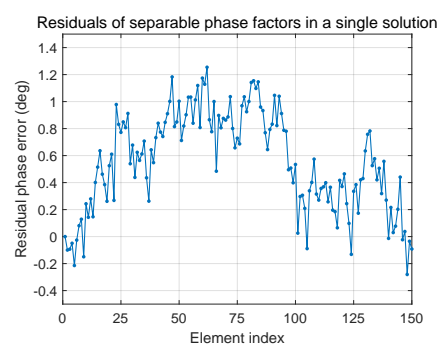

(a)

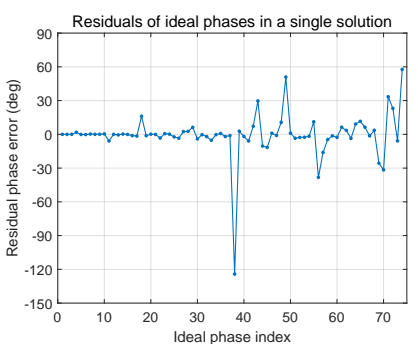

(b)
Fig. 7. Sample residual phase errors in a single solution, (a) separable phase factors, and (b) ideal phases. Results are obtained by using the initial guess shown in Fig. 5.

However, the residuals of the directly estimated ideal phases are still large for the low-SNR baselines. Fortunately, there is another approach to calibrate the ideal phase of each baseline by (15). As illustrated in Fig. 8, the estimation of the baseline-related phases shows a much better performance. Due to the triangular-shaped residual phase errors shown in the estimated separable phase factors, the baseline-related phase residual errors increase gradually with baseline index; and similar residual's variation can be found among the groups of same-length baselines. Although the maximum baselinerelated residual phase error is around $1.5^{\circ}$ in this example, it can be further reduced by implementing averaging methods.

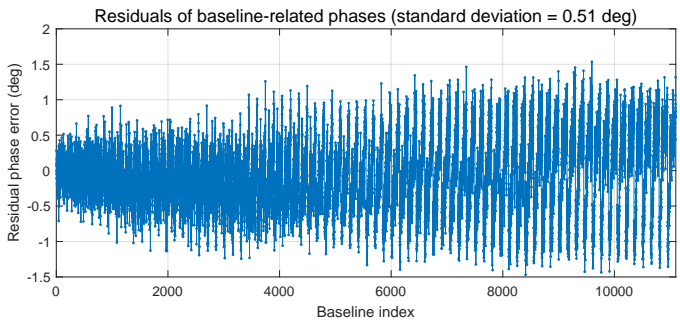

Fig. 8. Sample residuals of baseline-related phases in a single solution. The baseline index is formed by groups of redundant baselines in an ascending length order.

Consequently, the baseline-related phases are used to evaluate the phase calibration performance in the following analysis.

\section{Performance of the Phase Calibration with Averaging}

The performance of the phase calibration strategy is evaluated in the following aspects:

- Methods of averaging: For comparison, averaging of the solutions in a half array rotation and averaging of the complex measurements in a complete array rotation are simulated, respectively.

- Noise standard deviation: A wide range of noise level is simulated to evaluate its influence on the phase calibration. Although the simulations are conducted at the complex correlation level, the noise standard deviations 
shown in the figures are transformed into an equivalent denormalized visibility level with the system temperature of $700 \mathrm{~K}$ for better readability ${ }^{2}$.

- Array configuration: The total number of elements is always set to be even, in order to present the half-rotation calibration performance [1]. Different combinations of the total number of elements $M$ and adjacent element spacing $d_{s}$ are evaluated, which can be used as a reference for the system scalability.

\section{1) Phase Calibration Residuals:}

The standard deviation of the baseline-related residual phase error is shown in Fig. 9, where the residuals are calculated by (16). In both averaging cases, the residual's standard deviation at $\sigma_{\mathrm{I}, \mathrm{R}}=0 \mathrm{~K}$ is consistent with the predefined nonseparable phase factors $\left(\phi_{k l} \sim N\left(0,0.15^{2}\right) \mathrm{deg}\right)$. For the phase calibration conducted by a half array rotation, it works well with small adjacent element spacing. However, when a large antenna spacing is considered $\left(d_{s}=2 \lambda\right)$, the sampling density becomes sparser and the large-magnitude visibilities are less sampled. Thus, the phase calibration becomes susceptible to noise and fails to converge when $\sigma_{\mathrm{I}, \mathrm{R}} \geq 0.05 \mathrm{~K}$.

On the other hand, the phase calibration conducted by a complete array rotation is significantly robust not only to the noise but also to different array configurations.

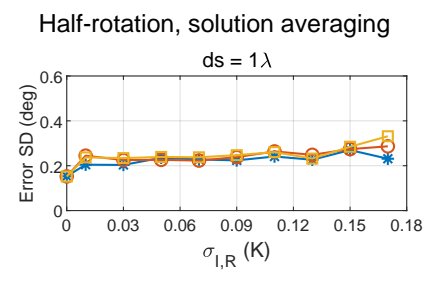

Full-rotation, measurements averaging
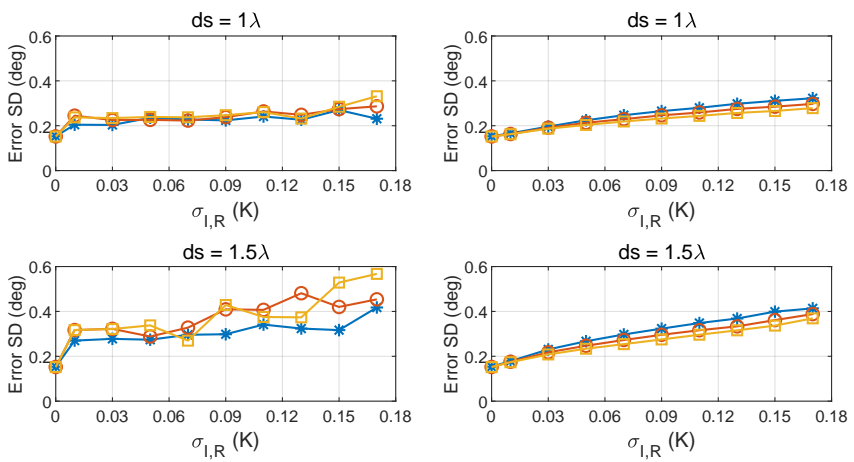

ds $=2 \lambda$
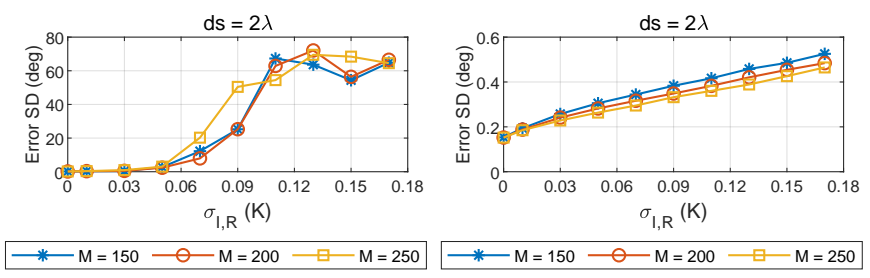

(a)

(b)

Fig. 9. Simulation results of phase calibration residuals, (a) averaging of the solutions in a half array rotation, and (b) averaging of the complex measurements in a complete array rotation. Note that the residual phase errors presented at $\sigma_{\mathrm{I}, \mathrm{R}}=0 \mathrm{~K}$ are obtained when only non-separable phase factors are considered (averaged of 300 Monte-Carlo runs).

Comparing the performances between these two averaging methods, averaging of the complex measurements is notably more stable, and the residual's standard deviation is less than $0.6^{\circ}$ even under very noisy circumstances. The improvement

\footnotetext{
${ }^{2} \mathrm{~A}$ typical noise standard deviation of visibility for rotational sampling strategy: $\sigma_{\mathrm{I}, \mathrm{R}}=0.1 \mathrm{~K}$, with $T_{\text {sys }}=700 \mathrm{~K}, B=300 \mathrm{MHz}, \tau=0.1 \mathrm{~s}$, three-level quantization, and $4 \mathrm{~B}$ sampling rate.
}

not only results from the two-fold measurements accomplished by a complete array rotation, but also mainly the significant noise reduction achieved by averaging of the complex measurements. Moreover, averaging of the complex measurements is much less time-consuming because the resolution of the system of phase calibration equations is conducted only once to obtain the final solution. Last but not least, the phase calibration with averaging of complex measurements shows a better performance with increasing the number of elements.

2) Image Reconstruction Errors:

Typical error maps are illustrated in Fig. 10 to observe the image reconstruction error caused by the phase calibration residuals, and the case that the non-separable phase factors are not included in the residuals is also presented for comparison. Apparently, the errors are concentrated on the edge of the Earth disk in both cases, where steep transition of the brightness temperature happens. Comparing the difference between these two cases, the error map is more noisy when the nonseparable phase factors are taken into account, since they are independently different among baselines. Also, the RMS error within the Earth disk is almost tripled, which implies that the non-separable phase factor is the main source of image reconstruction error after the phase calibration.

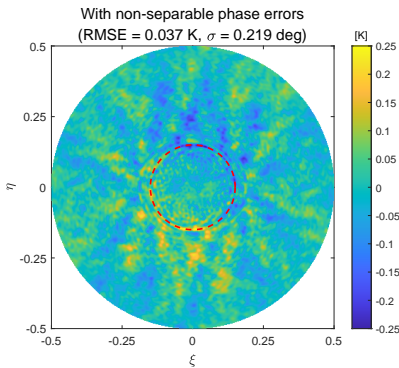

(a)

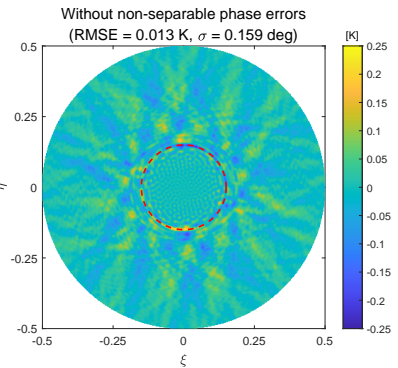

(b)
Fig. 10. Sample error maps of image reconstruction after phase calibration. (a) The non-separable phase factors are included in the phase residuals, (b) the non-separable phase factors are not included in the phase residuals. The red dot line indicates the Earth disk range $\xi^{2}+\eta^{2} \leq 0.15^{2}$.

The statistics of the RMS image reconstruction error caused by the phase calibration residuals are shown in Fig. 11, where averaging of the complex measurements in a complete array rotation is used as the phase calibration scheme. Even though the non-separable phase factors are unsolvable during the phase calibration, the RMS errors are less than $0.1 \mathrm{~K}$ even in the worst cases. And if the non-separable phase factors are not taken into account during image reconstruction, the RMS errors are reduced below $0.05 \mathrm{~K}$. In addition, the image reconstruction performance also takes advantage of the increase of element's quantity, as it has been found in the phase calibration residuals. The evaluation of the image reconstruction error further demonstrates that the proposed phase calibration strategy works robustly and precisely.

\section{Simulation Results of Amplitude Calibration}

The amplitude calibration based on redundant space calibration is a relative calibration, and an auxiliary calibration method is needed to provide any one of the system noise 

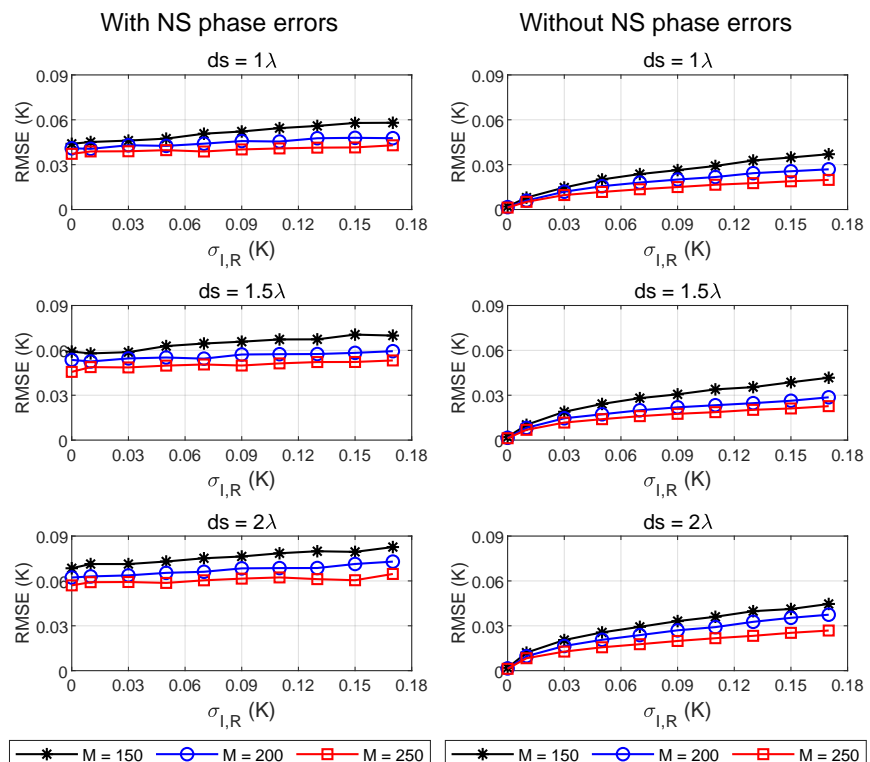

(a)

(b)

Fig. 11. RMS errors of image reconstruction caused by the phase calibration residuals, (a) the non-separable phase factors are included in phase residuals, and (b) the non-separable phase factors are not included in phase residuals (averaged of 300 Monte-Carlo runs).

temperatures to accomplish the absolute amplitude calibration (for example, the noise-injection calibration of at least one receiving channel). As the system temperatures obtained by the proposed amplitude calibration strategy are different from their true values by a multiplicative factor, the uncertainty of auxiliary calibration will introduce less than $0.2 \%$ error if an accurate calibration is implemented (if $\Delta T=1 \mathrm{~K}$ and $T_{\text {sys }}=700 \mathrm{~K}$, it will introduce $0.14 \%$ error in the absolute calibration of each element's system temperature). Without loss of generality, we assume that the reference element's system temperature is exactly provided in the following analysis.

Despite that the ideal amplitudes of each same-length baseline group can be obtained by solving the system of amplitude calibration equations, the ideal amplitude of every single baseline can also be estimated by

$$
\left|V_{k l}^{\mathrm{cal}}\right|=\frac{\left|V_{k l}^{\mathrm{raw}}\right|}{\hat{g}_{k} \cdot \hat{g}_{l}}
$$

where $\hat{g}_{k, l}$ are the estimated separable amplitude factors. This procedure is also denoted by the denormalization of visibility [19], but the non-separable amplitude factor is omitted here and left for the calibration residual. The ideal amplitude calculated by (17) is denoted as the baseline-related amplitude in the following analysis, in order to be distinguished from the directly estimated ideal amplitude. As the amplitude calibration model is described in a multiplicative relationship, it is more convenient to evaluate the amplitude calibration residual in percent error:

$$
\begin{aligned}
\delta_{k l}^{A} & =\frac{\left|V_{k l}^{\mathrm{cal}}\right|-\left|V_{k l}^{\mathrm{id}}\right|}{\left|V_{k l}^{\mathrm{id}}\right|} \cdot 100 \% \\
& =\frac{g_{k l} \cdot g_{k} \cdot g_{l}-\hat{g}_{k} \cdot \hat{g}_{l}}{\hat{g}_{k} \cdot \hat{g}_{l}} \cdot 100 \%
\end{aligned}
$$

where the non-separable amplitude factor $g_{k l}$ remains as the amplitude calibration residual.

\section{A. Single Solution}

The single solution discussed here has the same definition as we made for the phase calibration, and it is presented to show the general properties of solution.

A typical example of the estimated ideal amplitude residuals is shown in Fig. 12(a). Although the absolute residual errors are mostly less than $0.05 \mathrm{~K}$, it should be pointed out that the ideal amplitudes for long baselines are around $0.01 \mathrm{~K}$, and the percent errors are tremendously large for those low-SNR baselines.

Similar to the phase calibration, it is better to calibrate amplitude by the baseline-related amplitudes introduced in (17). A sample histogram of the percent errors of the baselinerelated amplitudes is shown in Fig. 12(b), and their mean value and standard deviation are denoted as the amplitude calibration bias and uncertainty, respectively. The percent errors including the non-separable amplitude factors have a standard deviation of $2.25 \%$, and the standard deviation is reduced to $0.97 \%$ when the non-separable amplitude factors are not included in the amplitude calibration residuals. Note that the non-separable amplitude factors are set to have a standard deviation of $2 \%$ in the simulation, so it acts as the main error source of the calibration uncertainty. On the other hand, it is obvious to notice that the amplitude calibration suffers from a calibration bias of $2.28 \%$ in both cases.

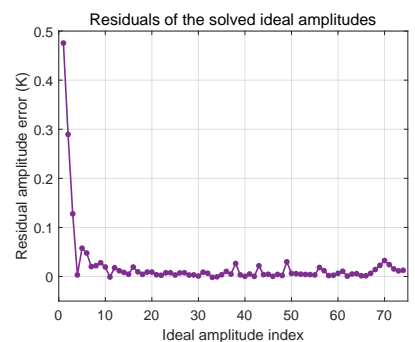

(a)

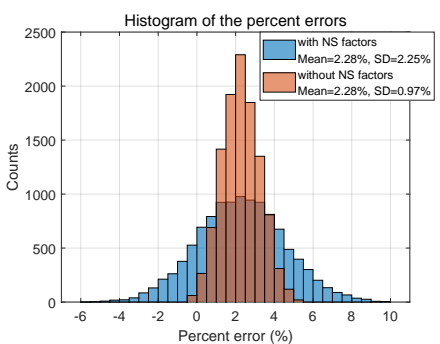

(b)
Fig. 12. Sample residual amplitude calibration errors in a single solution. (a) Residuals of the directly estimated ideal amplitudes. (b) Histogram of the percent errors of the baseline-related amplitudes. The simulation is conducted by an array with 150 elements, adjacent element spacing $d_{s}=1 \lambda$.

Fig. 13 shows the typical image reconstruction error maps, where the visibilities are calibrated by the baseline-related amplitudes using the same results presented in Fig. 12(b). The case that the non-separable amplitude factors are not taken into account is also presented for comparison. The image reconstruction bias within the Earth disk is clearly seen in both cases, and the error behaviors are different between the Earth disk and the cold sky, which will be demonstrated scenedependent in Section VI. The error map appears to be noisier when the non-separable amplitude factors are included in the calibration residuals. In addition, the RMS errors evaluated within the Earth disk do not change much whether the nonseparable amplitude factors are taken into account or not. 


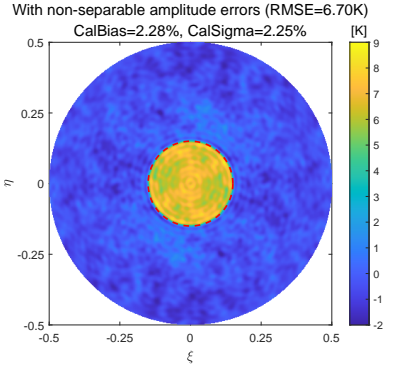

(a)

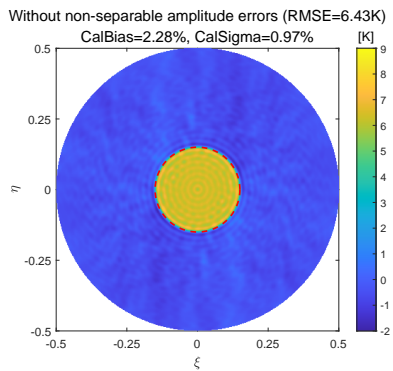

(b)
Fig. 13. Sample error maps of image reconstruction after the amplitude calibration. (a) The non-separable amplitude factors are included in the amplitude residuals, (b) the non-separable amplitude factors are not included in the amplitude residuals.

\section{B. Performance of the Amplitude Calibration with Averaging}

The performance of the amplitude calibration strategy is evaluated in the following aspects:

- Methods of averaging: Since weighted least-squares method has a property of linearity, averaging of solutions will produce identical results as the averaging of measurements, so it is omitted. For comparison, averagings of the measurements in both half and complete array rotation are simulated, respectively. Also, there are three kinds of averaging objects to be evaluated ${ }^{3}$.

- Noise standard deviation and array configuration: Same as the configuration settings for the phase calibration.

1) Amplitude Calibration Residuals:

The baseline-related amplitudes are used to evaluate amplitude calibration residuals in two aspects: 1) calibration bias, and 2) calibration uncertainty. Calibration bias has been found to have a zero-mean property among the Monte Carlo simulations, so the standard deviation of calibration biases is calculated to indicate its common range.

The bias and uncertainty of the amplitude calibration are shown in Fig. 14 and Fig. 15, respectively; where all kinds of averaging methods are presented for comparison. Different methods of averaging do not show significant difference in the calibration performance. Overall, averaging of the complex measurements in a complete array rotation is preferred to obtain the best calibration performance.

The calibration bias is found to be around $1.0 \%$ in each case, and it remains about $0.3 \%$ when thermal noise is not considered in the simulation $\left(\sigma_{\mathrm{I}, \mathrm{R}}=0 \mathrm{~K}\right)$. In fact, no weight should be applied when non-separable amplitude factors are the only measurement errors, because the non-separable amplitude factors are assumed to have the same standard deviation among different baselines ${ }^{4}$. To further clarify the reason for the amplitude calibration bias, the amplitude calibration is simulated when all non-separable amplitude factors are ideally equal to 1, and the results can be found in the Appendix C. It

\footnotetext{
${ }^{3}$ In the following figure legends, AmpMean denotes the averaging of the measured amplitudes, AmpLnMean denotes the averaging of the natural logarithm of the measured amplitudes, and CompMean denotes the averaging of the measured complex measurements [1].

${ }^{4}$ After taking the natural logarithm of the amplitude calibration model as shown in (12), it yields $\ln \left(g_{k l}\right) \sim N\left(0,0.02^{2}\right)$, where the following approximation is used: $\ln (1+x) \approx x$, if $x \approx 0$.
}

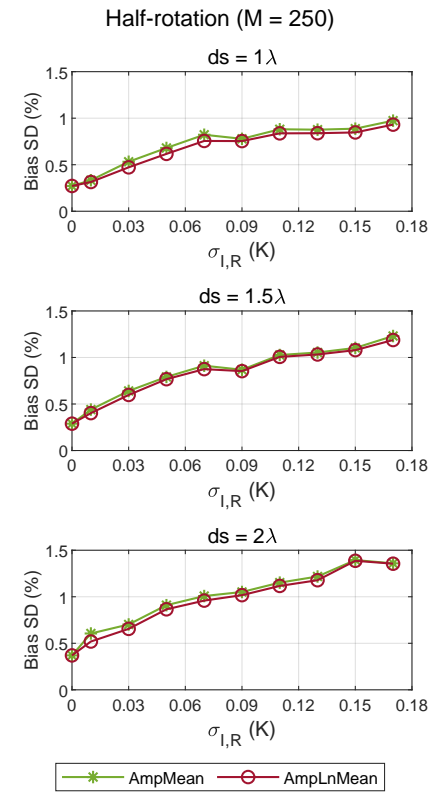

(a)
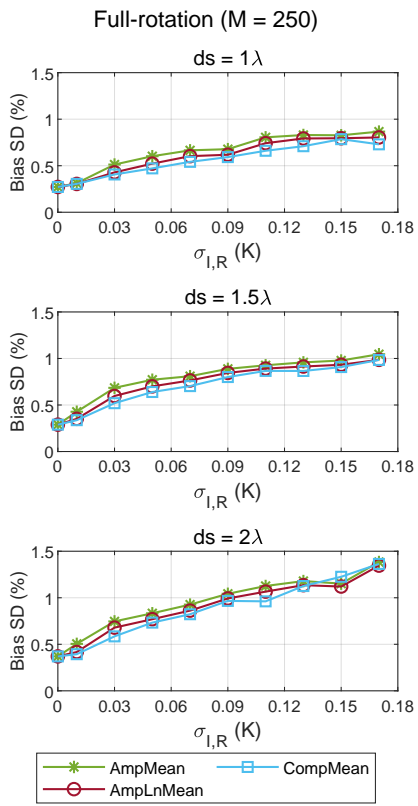

(b)
Fig. 14. Simulation results of the amplitude calibration bias, (a) half array rotation; (b) complete array rotation. The total number of elements $M$ is equal to 250 in each case (averaged of 300 Monte-Carlo runs).
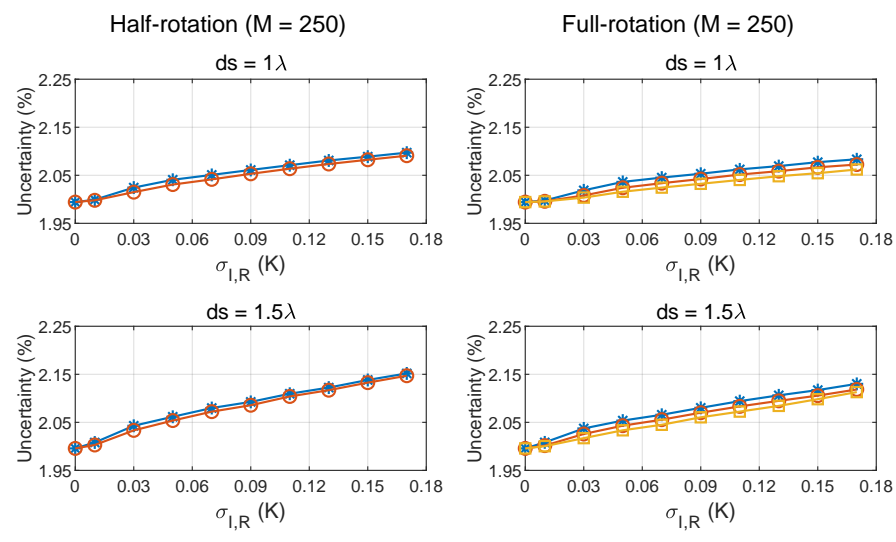

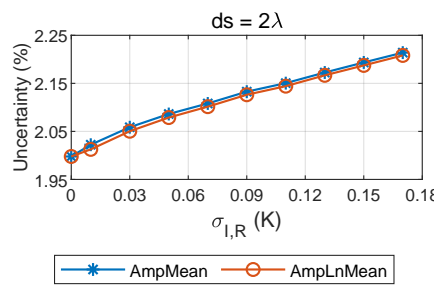

(a)

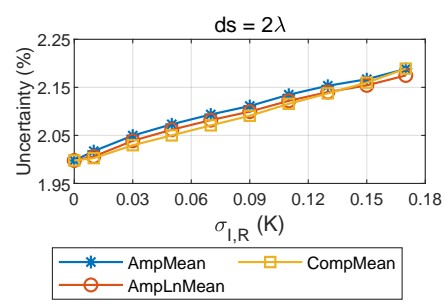

(b)
Fig. 15. Simulation results of the amplitude calibration uncertainty, (a) half array rotation; (b) complete array rotation. The total number of elements $M$ is equal to 250 in each case (averaged of 300 Monte-Carlo runs).

shows that the calibration bias also exists even when thermal noise is the only measurement error, but less than $1 \%$ in each case. As mentioned in [18], the redundant space calibration of visibility amplitude suffers from error amplification because the exponentiation is used in the de-linearization process. Besides, thermal noise will gradually introduce a constant bias in the natural logarithmic form of the measured amplitude, 
as we have found in Fig. 3. It can be recognized that the amplitude calibration bias is a combined outcome caused by both thermal noise and non-separable amplitude factor, also the side effects brought by logarithm and exponentiation.

The calibration uncertainty floor at $\sigma_{\mathrm{I}, \mathrm{R}}=0 \mathrm{~K}$ is consistent with the predefined non-separable amplitude factors $\left(g_{k l} \sim\right.$ $N\left(1,0.02^{2}\right)$ ), and the calibration uncertainty grows gradually with respect to the noise level. The main contributions to the calibration uncertainty come from the non-separable amplitude factors. As presented in the Appendix C, the calibration uncertainty caused by the misfit of the estimated separable amplitude factors is less than $1 \%$ (when the non-separable amplitude factors are not included in the amplitude calibration residuals).

Similarly, the amplitude calibration also shows a better performance with the increase of the total number of elements, but only for the calibration uncertainty, and no apparent improvement can be identified for the calibration bias. The detailed comparison of the amplitude calibration residuals with different element's quantity can be found in the Appendix C.

2) Image Reconstruction Errors:

The statistics of RMS image reconstruction error caused by the amplitude calibration residuals are shown in Fig. 16, where averaging of the complex measurements in a complete array rotation is used as the amplitude calibration scheme. A considerable image reconstruction error around $2 \mathrm{~K}$ has been found in each case, and no significant improvement can be seen when the non-separable amplitude factors are not included in the amplitude residuals. As the non-separable amplitude factor is a zero-mean random variable, it will only introduce calibration uncertainty when the amplitude residuals are evaluated. Thus, the image reconstruction error is mainly contributed by the amplitude calibration bias. Note that the statistics shown in Fig. 16 can only be applied to the case that the original brightness temperature is equal to $300 \mathrm{~K}$.

Clearly, the amplitude calibration residuals will introduce a non-negligible image reconstruction error, primarily due to the amplitude calibration bias. Further study on the image reconstruction error caused by the amplitude calibration bias and its correction are presented in Section VI.

\section{Study on Amplitude CALIBRATion BiAs AND ITS CORRECTION}

\section{A. The Origin of the Image Reconstruction Bias}

To investigate the reason for the image reconstruction bias illustrated in Fig. 13, a typical example of the percent errors of baseline-related amplitudes is examined from the perspective of the same-length baseline groups, as shown in Fig. 17(a), and their mean values are shown in Fig. 17(b). Although the percent errors of each baseline are quite different, their mean values along the same-length baseline groups are almost identical.

Considering a most general case, if we assume that the non-zero baseline visibilities are influenced by a constant multiplicative factor $\left(1+\alpha_{A}\right)$ simultaneously ${ }^{5}$, the image

\footnotetext{
${ }^{5}$ The factor $\alpha_{A}$ has the same definition of the amplitude calibration bias presented in Section V. The zero-baseline visibility remains as its ideal value, since it is not affected by the amplitude calibration bias.
}
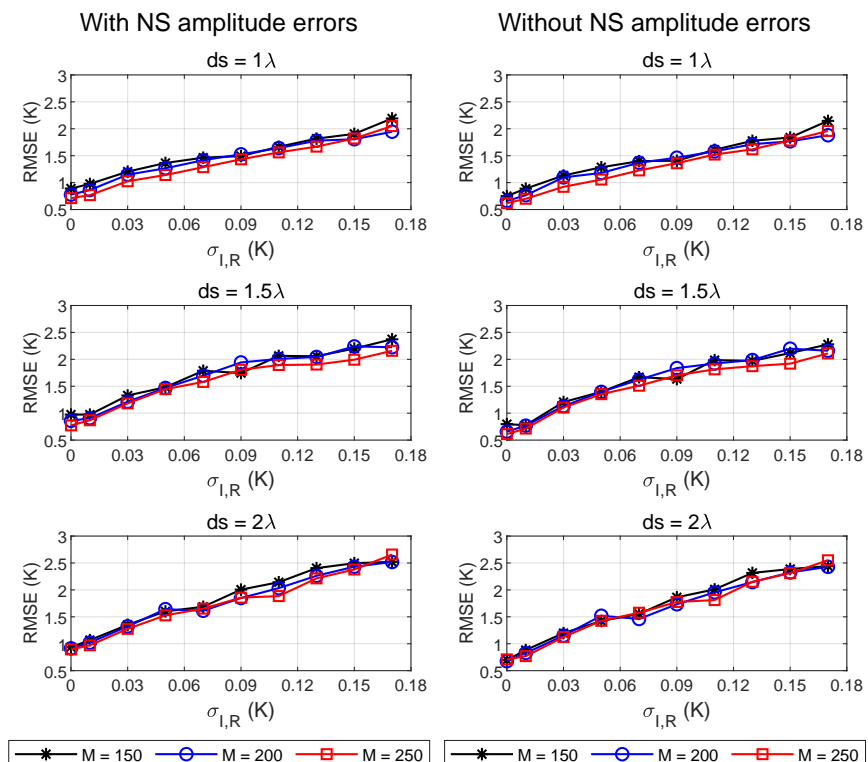

(a)

(b)

Fig. 16. RMS errors of image reconstruction caused by the amplitude calibration residuals, (a) the non-separable amplitude factors are included in amplitude residuals, and (b) the non-separable amplitude factors are not included in amplitude residuals (averaged of 300 Monte-Carlo runs).

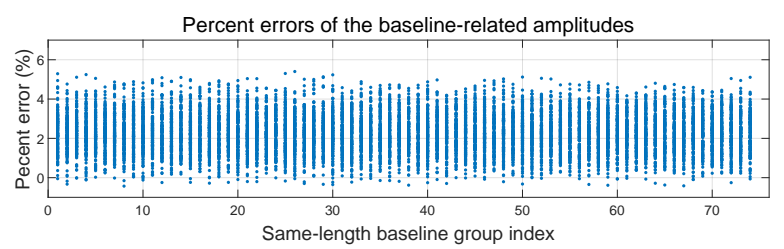

(a)

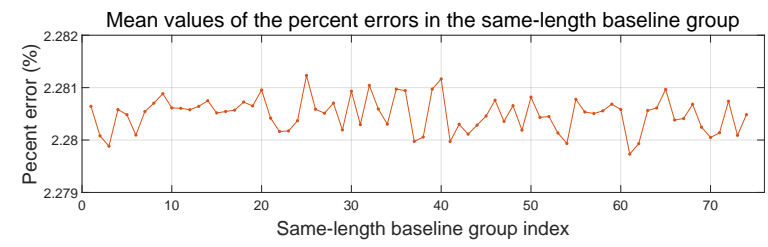

(b)

Fig. 17. Sample percent errors of the baseline-related amplitudes, (a) organized in groups of same-length baselines. (b) The mean percent error along each group of same-length baselines. The data shown here are the same as those shown in Fig. 12(b).

reconstruction of the corresponding multiplicatively biased visibilities can be written by

$$
\begin{aligned}
\hat{T}_{B}^{\mathrm{bias}}(\xi, \eta)= & \mathcal{F}^{-1}\left[\left(1+\alpha_{A}\right) V_{k l}\right]+\mathcal{F}^{-1}\left[V_{0} \cdot \delta(u, v)\right] \\
= & \mathcal{F}^{-1}\left[\left(1+\alpha_{A}\right) V_{k l}\right]+\mathcal{F}^{-1}\left[\left(1+\alpha_{A}\right) V_{0} \cdot \delta(u, v)\right] \\
& -\mathcal{F}^{-1}\left[\alpha_{A} V_{0} \cdot \delta(u, v)\right] \\
= & \left(1+\alpha_{A}\right) \cdot T_{B}^{\mathrm{ref}}(\xi, \eta)-\alpha_{A} V_{0}
\end{aligned}
$$

where $\mathcal{F}^{-1}[\cdot]$ is the inverse Fourier transform operator (it can be generalized to any specific image reconstruction method), $V_{k l}$ is the nonzero-baseline visibility, $V_{0}=V(0,0)$ is the zero-baseline visibility, and $T_{B}^{\mathrm{ref}}(\xi, \eta)$ is the original brightness temperature. Note that the relationship $T_{B}^{\mathrm{ref}}(\xi, \eta)=$ 


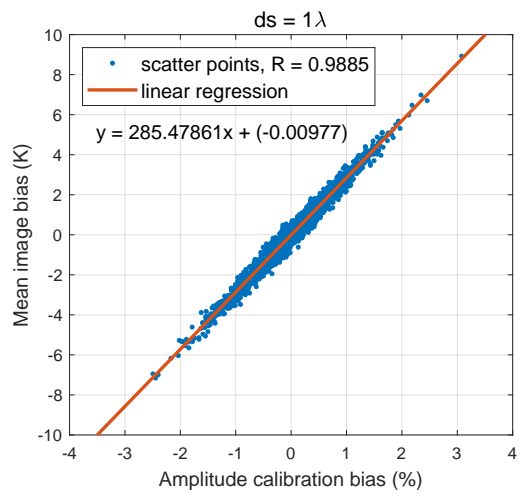

(a)

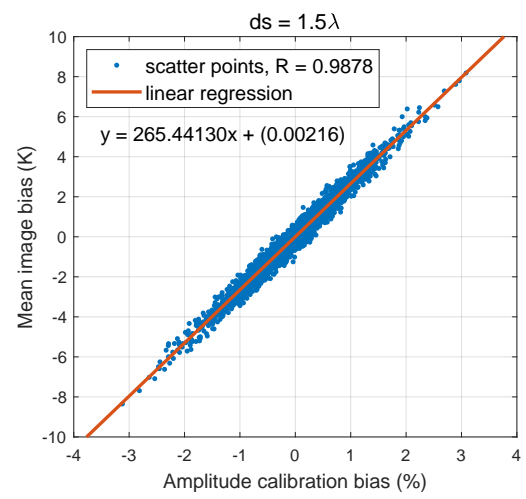

(b)

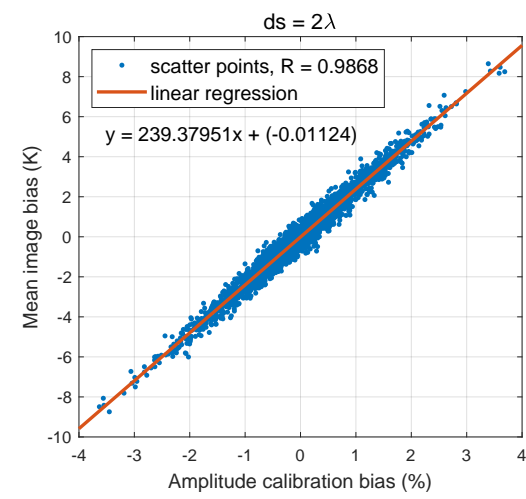

(c)

Fig. 18. Mean image reconstruction bias versus amplitude calibration bias, (a) $d_{s}=1 \lambda$, (b) $d_{s}=1.5 \lambda$, (c) $d_{s}=2 \lambda$. The image bias is evaluated within the Earth disk, and the amplitude calibration is conducted by averaging of the complex measurements in a complete array rotation.

$\mathcal{F}^{-1}\left[V_{k l}+V_{0} \cdot \delta(u, v)\right]$, and the linearity of inverse Fourier transform have been used during the derivation.

Moreover, the corresponding image reconstruction error (bias) can be evaluated by

$$
\begin{aligned}
\epsilon_{T}(\xi, \eta) & =\hat{T}_{B}^{\mathrm{bias}}(\xi, \eta)-T_{B}^{\mathrm{ref}}(\xi, \eta) \\
& =\alpha_{A}\left[T_{B}^{\mathrm{ref}}(\xi, \eta)-V_{0}\right] .
\end{aligned}
$$

Thus, the image reconstruction bias of each specific position $\epsilon_{T}(\xi, \eta)$ is found to have the linear relationship with respect to the original brightness temperature $T_{B}^{\mathrm{ref}}(\xi, \eta)$ (the so-called scene-dependent bias) and the constant amplitude bias $\alpha_{A}$.

\section{B. Validation}

Recalling that we have found the image reconstruction bias caused by the amplitude calibration residuals have different error behavior between the Earth disk and the cold sky, it is valuable to verify whether it obeys the relationship presented in (20).

Fig. 18 shows the mean image reconstruction bias within the Earth disk versus its corresponding amplitude calibration bias, and the data are collected from the Monte Carlo simulations of the amplitude calibration presented in Section V-B. The mean image reconstruction bias shows a significant linearity with respect to the amplitude calibration bias. Moreover, the corresponding intercepts are small enough to be omitted, which agrees with the linear relationship presented in (20).

Although the actual amplitude calibration biases are not identical on every single baseline (see Fig. 12(b) and Fig. 17(a)), the resulting image reconstruction biases show the same property when the amplitude calibration bias is a constant for each baseline. It can be somehow explained by the fact that the averaged amplitude calibration bias is almost identical among each same-length baseline group. In addition, the visibilities are averaged by a redundancy factor of 2 before image reconstruction, which further decreases the dispersion of the amplitude calibration biases.

However, the theoretical value of the slope ${ }^{6}$ inferred by (20) should be $T_{B}^{\mathrm{ref}}-V_{0}=300-21.2=278.8 \mathrm{~K} / \mathrm{K}$, and the

\footnotetext{
${ }^{6} \mathrm{We}$ have verified that if uniformly-sampled Cartesian $u$ - $v$ grids and ordinary IFFT are used, the fitted slope perfectly matches with the theoretical value.
}

slopes obtained by the linear regressions in Fig. 18 are quite different from this value. This phenomenon can be attributed to the non-linearity introduced by the IPPFFT, where the interpolations are used to fit the circularly sampled visibilities into the pseudo-polar grids. The theoretical slope for IPPFFT can be estimated by performing an ideal image reconstruction of the multiplicatively biased visibilities with a preset constant amplitude bias $\alpha_{A}$ :

$$
\begin{aligned}
s & =\frac{\operatorname{IPPFFT}\left[\left(1+\alpha_{A}\right) V_{k l}+V_{0}\right]-\operatorname{IPPFFT}\left[V_{k l}+V_{0}\right]}{\alpha_{A}} \\
& =\frac{\epsilon_{T}^{\prime}}{\alpha_{A}}
\end{aligned}
$$

and a series of amplitude biases $\alpha_{A}$ and its corresponding image errors $\epsilon_{T}^{\prime}$ can be gathered to estimate the theoretical slope more precisely by a linear regression fit.

Table I summarizes the slopes regressed from the amplitude calibration results and their theoretical values estimated by (21). A great consistency between the Monte-Carlo simulation results and the theoretical values can be found, which demonstrates that the relationship presented in (20) is also valid for IPPFFT.

TABLE I

COMPARISON OF THE SLOPES FOR IPPFFT OBTAINED BY MONTE-CARLo RESUltS AND THEORETICAL VAlues

\begin{tabular}{lcc}
\hline \hline & Monte-Carlo result & Theoretical value \\
\hline$d_{s}=1 \lambda$ & 285.4786 & 285.5139 \\
$d_{s}=1.5 \lambda$ & 265.4413 & 265.3382 \\
$d_{s}=2 \lambda$ & 239.3795 & 239.2336 \\
\hline
\end{tabular}

\section{Correction of the Image Reconstruction Bias}

The analysis above introduces a potential method to estimate the amplitude calibration bias that inherently existed in redundant space calibration. We have found that the calibration bias is strongly related to the specific values of non-separable amplitude factors, and it remains stable if there is not a significant variation of the non-separable amplitude factors and system noise temperatures. Since the zero-baseline visibility 


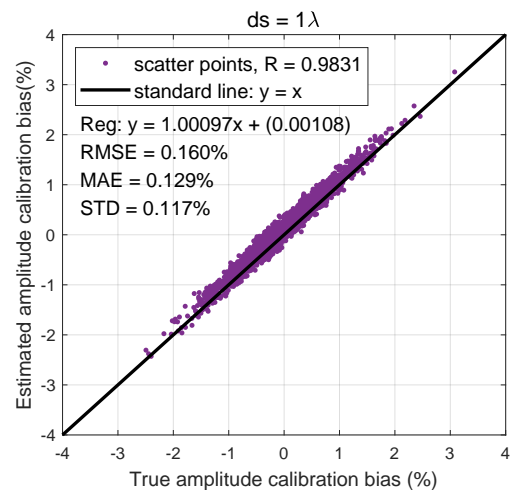

(a)

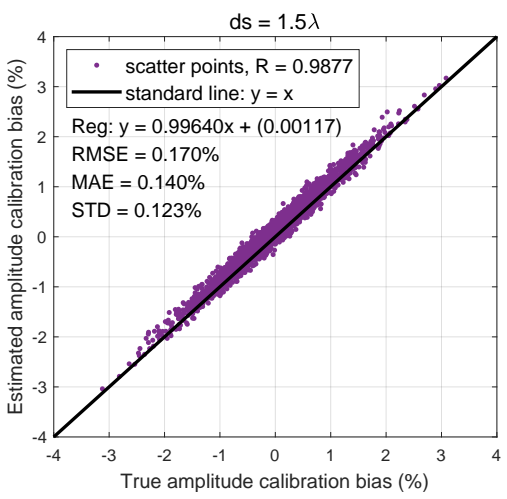

(b)

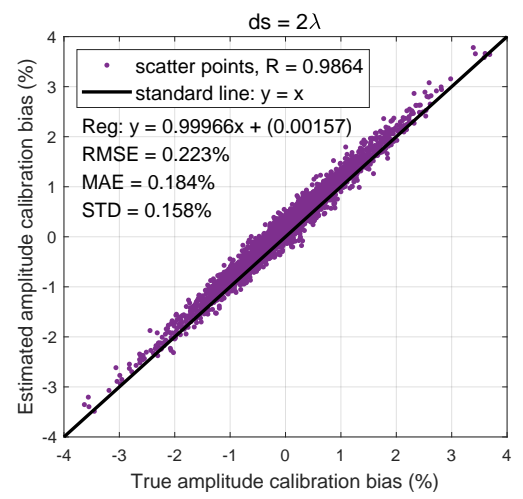

(c)

Fig. 19. Amplitude calibration bias versus its estimation, (a) $d_{s}=1 \lambda$, (b) $d_{s}=1.5 \lambda$, (c) $d_{s}=2 \lambda$. The black line presents the standard line $\mathrm{y}=\mathrm{x}$.

$V_{0}$ is provided by the total-power radiometers (such as noiseinjection radiometer), the amplitude calibration bias can be estimated if an a priori brightness temperature of a region within the observation scene $T_{B}^{\text {pri }}(\xi, \eta)$ is provided:

$$
\hat{\alpha}_{A}^{\prime}=\frac{\hat{T}_{B}^{\text {bias }}(\xi, \eta)-T_{B}^{\mathrm{pri}}(\xi, \eta)}{s}
$$

where $\hat{\alpha}_{A}^{\prime}$ is the estimated amplitude calibration bias, and $s$ is the slope we obtained by (21) for the specific array configuration. Note that the measurement of the zero-baseline visibility also has an error term $\hat{V}_{0}=V_{0}+\Delta V_{0}$, and this error term can be canceled out if two regions with a priori brightness temperatures are provided.

To verify the estimation of the amplitude calibration bias, we assumed that there is a region within the $300 \mathrm{~K}$ Earth disk $\left(0.01 \leq r \leq 0.03,0^{\circ} \leq \phi \leq 45^{\circ}\right)$ whose brightness temperature is a priori known, and the reconstructed brightness temperatures after amplitude calibration within this area are averaged to conduct estimation by using (22).

Fig. 19 shows the resulting estimation of the amplitude calibration bias versus its true value, the data are computed from the Monte Carlo simulations of the amplitude calibration presented in Section V-B. It is clear that a good estimation has been accomplished. Overall, the RMS errors of each case are less than $0.25 \%$, and the mean absolute errors are less than $0.20 \%$. However, it should be pointed out that the estimation performance degrades slightly with the increase of adjacent element spacing, due to the image reconstruction error introduced by sparser spectrum sampling. Note that the reconstructed image used as $\hat{T}_{B}^{\text {bias }}$ is corrupted by thermal noise to simulate the realistic circumstance of bias estimation. Nevertheless, the thermal noise can reduced to a small level by the averaging of measured visibilities, since the visibilities have been sampled $M$ (or $M / 2$ ) times to conduct calibration.

Accordingly, one more step for the correction of the amplitude calibration bias is added into the amplitude calibration procedure

$$
\left|V_{k l}^{\mathrm{cal}}\right|=\frac{\left|V_{k l}^{\mathrm{raw}}\right|}{\hat{g}_{k} \cdot \hat{g}_{l} \cdot\left(1+\hat{\alpha}_{A}^{\prime}\right)}
$$

where $\hat{\alpha}_{A}^{\prime}$ is the amplitude calibration bias estimated by (22).

Fig. 20(a) shows the statistics of the RMS image reconstruction errors after the amplitude calibration bias is corrected by the proposed method, where averaging of the complex measurements in a complete array rotation is used as the amplitude calibration scheme. As compared to the results presented in Fig. 16, the image reconstruction performance is notably improved, and the RMS errors are reduced to less than $0.65 \mathrm{~K}$ in each case. The amplitude calibration residuals at the current stage can be described in three aspects:
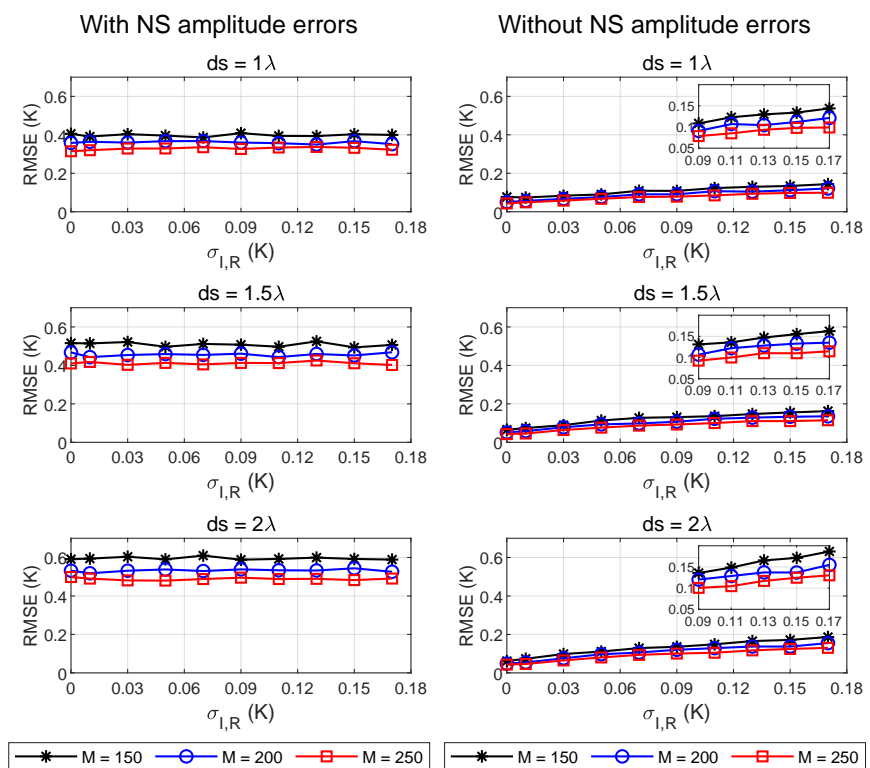

(a)

(b)

Fig. 20. RMS errors of image reconstruction after the amplitude calibration bias is corrected, (a) the non-separable amplitude factors are included in amplitude residuals, and (b) the non-separable amplitude factors are not included in amplitude residuals (averaged of 300 Monte-Carlo runs).

1) The residual amplitude calibration bias. As shown in Fig. 19 , the bias is not exactly corrected, but most of the residual biases have been alleviated to below $0.3 \%$;

2) The calibration uncertainty contributed by the misfit of the estimated separable amplitude factors, less than $1 \%$;

3) The calibration uncertainty contributed by the nonseparable amplitude factors, equal to $2 \%$.

The case that the non-separable amplitude factors are not included in the amplitude calibration residuals is also pre- 
sented in Fig. 20(b). Comparing these two cases, it is obvious that the non-separable amplitude factors become the main image reconstruction error source after the bias correction. Although the residual bias still exists, its contribution to image reconstruction error is less than the non-separable amplitude factors. Another point that needs to be mentioned is that the image reconstruction performance takes advantage of the increase of element's quantity after the amplitude calibration bias is effectively corrected.

\section{DISCUSSION}

Following the simulation results described above, some important results are discussed as below.

\section{A. General Calibration Performance}

Several calibration schemes have been presented and analyzed. In order to achieve the best calibration performance and minimum computational cost, the calibration should be conducted by averaging of the complex measurements accomplished by an integer number of revolution of array rotation, for both phase and amplitude calibration.

Although redundant space calibration is reported to have the problem of error amplification [18], the calibration strategy proposed in this paper alleviates this drawback significantly. Thanks to the closed structure of equispaced circular array and the massive baseline redundancy obtained by the array rotation, strong restrictions and self-consistencies are provided to the system of calibration equations. It is extremely important for the successful implementation of the proposed calibration strategy, especially when the system status is completely unknown without any a priori information.

The non-separable factors are the main calibration residual errors in the proposed calibration strategy because they cannot be solved by redundant space calibration. However, their contributions are inherently restricted by the Hermitian conjugate redundancy accomplished by the equispaced circular array with an even number of elements. Moreover, as a great deal of measurement redundancy is achieved by array rotation, a potential way to alleviate the effects of non-separable factors in the image reconstruction is averaging all the redundant measurements among the group of same-length baselines after the calibration.

Both phase and amplitude calibration performances show improvement by increasing the number of antennas, no matter in the calibration residual or in the image reconstruction. This feature makes the proposed calibration strategy suitable for the high spatial resolution applications accomplished by the large-scale array.

\section{B. Amplitude Calibration Bias}

A non-negligible calibration bias is found in the amplitude calibration during the simulations. The calibration bias further introduces a scene-dependent bias in the image reconstruction, which becomes the main drawback of the proposed amplitude calibration strategy. This bias exists inherently and unavoidably due to the amplifying effect introduced by the exponentiation during the de-linearization process, also the constantly positive noise bias after taking the natural logarithm of the measured amplitudes. Fortunately, the amplitude calibration bias turns out to share the nearly equivalent property with the constant multiplicative bias on each baseline, and a corresponding bias correction method is proposed. The bias correction requires a priori information of the scene's brightness temperature, but only a flat region is needed. After the effective bias correction, the image reconstruction error can be reduced to an acceptable level $(\sim 0.65 \mathrm{~K})$, and it is dominated by the contributions from the non-separable amplitude factors.

The bias correction's efficiency depends on the area of this $a$ priori region, since the reconstructed brightness temperatures need to be averaged to reduce the random errors introduced by other calibration residuals. Note that the brightness temperatures are smooth and homogeneous at higher bands in 50-56 $\mathrm{GHz}$ range [20], due to the high absorption of the Earth's atmosphere, which especially meets the requirement asked by the bias correction method. And an a priori brightness temperature can be provided more easily by either numerical weather models or actual measurements from polar-orbiting satellites. The hardware structure can be designed accordingly by using the wide-band front-end filter before down conversion, and then splitting each frequency band by different frequency selective filters. In this way, the difference of the non-separable complex factors among different frequency bands can be minimized. Therefore, the amplitude calibration bias estimated for the higher frequency bands can also be applied to the lower frequency bands, since the specific value of the amplitude calibration bias is strongly dependent on the non-separable amplitude factors.

\section{Extension to a Realistic Observation Scene}

The assumptions and the $300-\mathrm{K}$ flat Earth disk configured in Section II are designed to provide an intuitive sense of the proposed calibration performance, and especially facilitate the evaluation of image reconstruction errors. The brightness temperature from a geostationary orbit at $50.3 \mathrm{GHz}$ is modeled by the dedicated simulation system [21] to validate the proposed calibration strategy in a more realistic way.

In order to compare fairly with the simulation results presented previously, the simulation assumptions are kept, but the brightness temperature is replaced by the modeled one at $50.3 \mathrm{GHz}$. Besides, the directional antenna is also considered in another simulation setting by using the corrugated horn antenna implemented in GIMS-II [22], whose directivity is about $24 \mathrm{~dB}$. In this case, only the effect of spatial decorrelation and the difference between antenna patterns are omitted; and the brightness temperature is amplified by the reciprocal of antenna solid angle, then tapered by the antenna pattern. The observation scenes in these two newly added cases are illustrated in Fig. 21.

These extended simulations are conducted by averaging the complex measurements in a complete array rotation for both phase and amplitude calibration, and $d_{s}=2 \lambda$. The calibration performances are evaluated with the non-separable factors taken into account; and the results are illustrated in Fig. 22, 


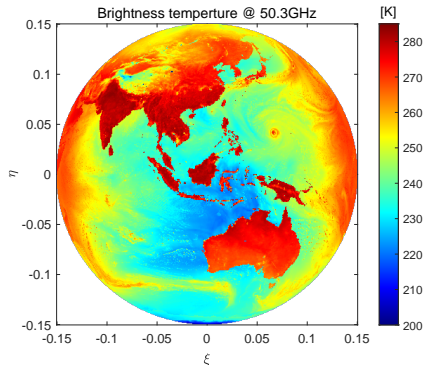

(a)

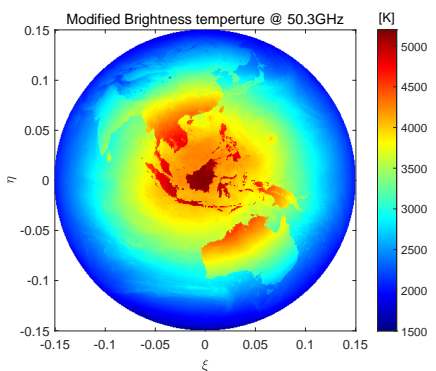

(b)
Fig. 21. Realistic observation scenes at $50.3 \mathrm{GHz}$, (a) isotropic antenna, (b) directional antenna.

Fig. 23, and Fig. 24, where the results obtained from the 300-K flat Earth disk are also presented for comparison.

When the isotropic antenna is considered, the calibration performances between the 300-K flat Earth disk and the brightness temperature at $50.3 \mathrm{GHz}$ are comparable, but a slight deterioration can be found. That is because the spatial frequency components are not that concentrated in the lowfrequency part when a natural scene is considered, especially for those observing frequencies in the window region. This property further reduces the SNR of the short baselines.

Furthermore, as the visibility amplitude is magnified by the antenna directivity when the directional antenna is considered, the calibration performance becomes more robust to noise corruption. The residual phase error is reduced to less than $0.2^{\circ}$ for phase calibration. The amplitude calibration bias is reduced to less than $0.8 \%$, and the amplitude calibration uncertainty is reduced to less $2.05 \%$.

Overall, the proposed calibration performance does not degenerate by enriching observation textures contributed from the atmosphere and underlying surfaces. The error floor (at $\sigma_{\mathrm{I}, \mathrm{R}}=0 \mathrm{~K}$ ) in these three cases is almost identical, which implies the ideally attainable calibration performance. It should be pointed out that the scene evolution is not considered in the simulations, which may introduce imperfect redundancies obtained by the array rotation. The calibration strategy may suffer from it, especially at $183-\mathrm{GHz}$ frequency bands where a rapid evolution of water vapor may occur. The dedicated research of this topic is left for our future work.

\section{The State of the Art of Other Reported Techniques}

Several techniques have been developed to calibrate other instruments with minimal implementation of noise-injection hardware, but each of them is based on different array configurations and auxiliary tools. Although a fair comparison is impossible, their performances are summarized to provide a reference of the state of the art.

A calibration scheme was proposed for the multisatellite interferometers with microwave beacons, where the antenna positions and separable phase factors can be calibrated simultaneously [23]. With four far-field beacons, the residual phase error is less than $0.5^{\circ}$ (standard deviation), and it can be further improved when near-field beacons could be implemented on orbit.
A hybrid calibration method for the Y-shaped array was proposed to combine redundant space calibration and reduced noise injection, in order to minimize system complexity of the noise-injection hardware [24]. Since array rotation is not taken into account, the calibration performance still depends on the noise-injection coverage and the SNR of visibility measurement. With a minimal coverage of $5 \%$, the residual phase error is around $2^{\circ}$ (visibility SNR $>25 \mathrm{~dB}$, RMS error), and the residual amplitude error is around $1 \%$ (visibility SNR $>25 \mathrm{~dB}$, RMS error).

Phase calibration methods based on the array rotation were proposed for Y-shaped array [25] and circular array [26], respectively. The core idea is to use the Hermitian conjugate symmetry inherently existed in interferometric measurements, but phase unwrapping technique is needed to solve the phase ambiguity. The residual phase error is less than $1.5^{\circ}$ for $\mathrm{Y}$ shaped array (visibility SNR $>30 \mathrm{~dB}$, RMS error), and less than $0.9^{\circ}$ for circular array (standard deviation).

\section{CONCLUSION}

In this paper, a comprehensive set of simulation and analysis of the proposed phase and amplitude calibration strategy have been presented.

The noise analysis indicates that the additive noise terms on the measured complex correlation have different behavior on the measured phase and amplitude, and the corresponding weighting functions are designed to mitigate it based on the noise variances.

The phase calibration strategy is demonstrated to have great precision and robustness. The residual phase error is less than $0.6^{\circ}$ (error standard deviation) even under the strongly noisy cases (up to $\sigma_{\mathrm{I}, \mathrm{R}}=0.17 \mathrm{~K}$ ), and the calibration performance does not degenerate when a high level of non-separable phase factors are considered (up to $\sigma_{\phi_{k l}}=1^{\circ}$, see Appendix D). Moreover, the RMS image reconstruction error caused by the phase calibration residual is less than $0.1 \mathrm{~K}$.

On the other hand, the amplitude calibration strategy turns out to have a calibration bias of $1 \%$, and a calibration uncertainty of $2 \%$ (dominated by the unsolvable non-separable amplitude factors). The resulting image reconstruction performance is corrupted by a scene-dependent bias. Accordingly, a bias correction method is proposed to be added into the amplitude calibration procedure, which requires an a priori regional brightness temperature within the observation scene. After the amplitude calibration bias is effectively corrected to less than $0.3 \%$, the RMS image reconstruction error caused by the amplitude calibration residual is less than $0.65 \mathrm{~K}$.

The proposed calibration strategy achieves a comparable performance with those techniques having the dedicated calibration hardware involved. The extended simulations of a realistic observation scene implies that a better calibration performance can be achieved by using the high-directivity antenna. This two-part paper has presented a preliminary study on the self-calibration of synthetic aperture interferometric radiometers, and it also provides an alternative calibration solution for the future geostationary microwave interferometric radiometers. 


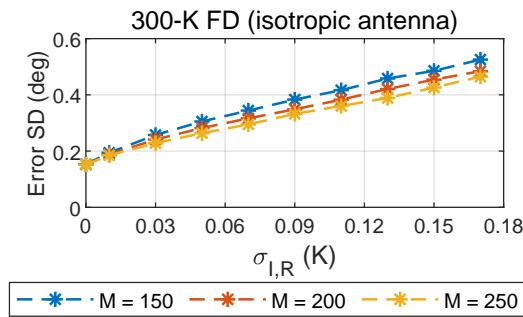

(a)

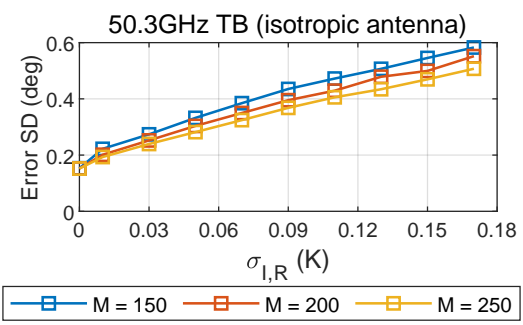

(b)

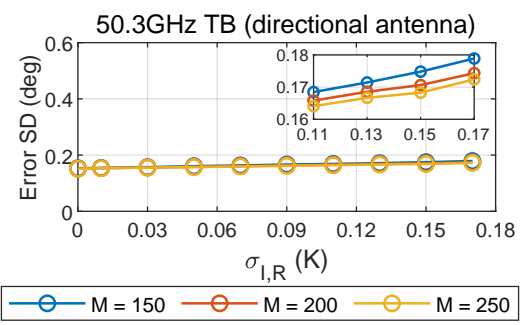

(c)

Fig. 22. Simulation results of the phase calibration residuals under different observation scenes, (a) 300-K flat Earth disk (isotropic antenna), (b) brightness temperature at $50.3 \mathrm{GHz}$ (isotropic antenna), (c) brightness temperature at $50.3 \mathrm{GHz}$ (directional antenna).

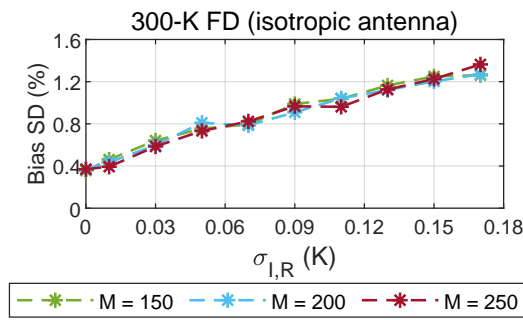

(a)

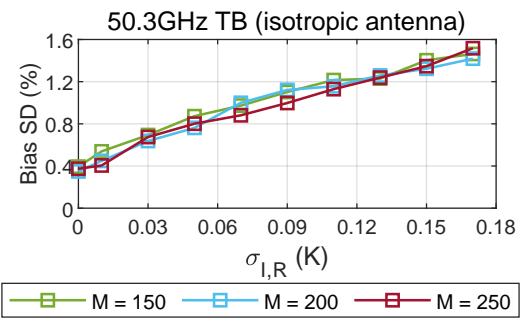

(b)

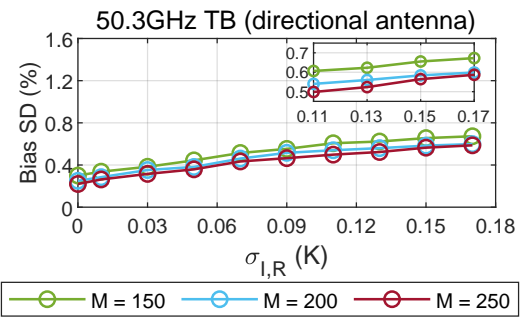

(c)

Fig. 23. Simulation results of the amplitude calibration bias under different observation scenes, (a) 300-K flat Earth disk (isotropic antenna), (b) brightness temperature at $50.3 \mathrm{GHz}$ (isotropic antenna), (c) brightness temperature at $50.3 \mathrm{GHz}$ (directional antenna).

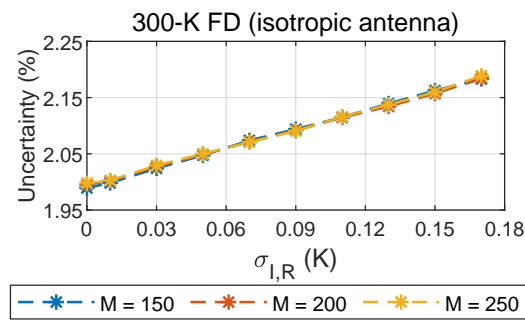

(a)

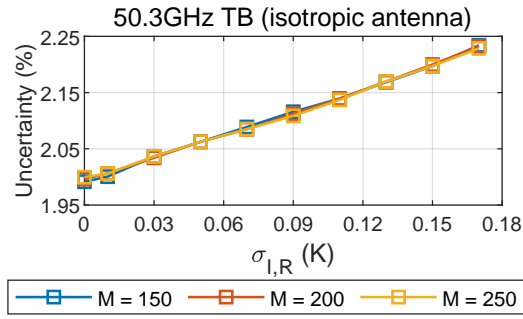

(b)

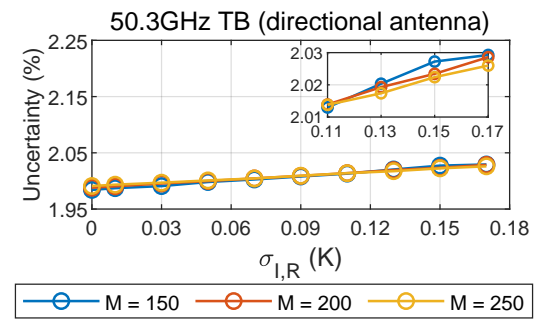

(c)

Fig. 24. Simulation results of the amplitude calibration uncertainty under different observation scenes, (a) 300-K flat Earth disk (isotropic antenna), (b) brightness temperature at $50.3 \mathrm{GHz}$ (isotropic antenna), (c) brightness temperature at $50.3 \mathrm{GHz}$ (directional antenna).

\section{APPENDiX A \\ VisibiLITY FUnCTION OF THE FLAT EARTH Disk}

Under the assumptions made in Section II, the relationship between the modified brightness temperature and the visibility function can be described as

$$
V(u, v)=\iint_{\xi^{2}+\eta^{2} \leq 1} T^{\prime}(\xi, \eta) \cdot e^{-j 2 \pi(u \xi+v \eta)} d \xi d \eta
$$

and the modified brightness temperature is

$$
T^{\prime}(\xi, \eta)=300 \mathrm{~K}, \quad \xi^{2}+\eta^{2} \leq 0.15
$$

Since the modified brightness temperature is centrosymmetric, and the visibility is sampled by a circular array, it is convenient to describe both domains in polar coordinates. Thus, (A-1) can be rewritten in polar coordinates as

$$
V\left(\rho, \theta_{s}\right)=\int_{0}^{2 \pi} \int_{0}^{1} T^{\prime}(r, \phi) \cdot e^{-j 2 \pi \rho \cdot r \cdot \cos \left(\theta_{s}-\phi\right)} r d r d \phi
$$

and (A-2) is rewritten as

$$
T^{\prime}(r, \phi)=T^{\prime}(r)=300 \mathrm{~K}, \quad 0 \leq r \leq 0.15 .
$$

Using the integral representation of zero-order Bessel Functions of the first kind

$$
J_{0}(z)=\frac{1}{2 \pi} \int_{0}^{2 \pi} e^{j z \cos (\theta-\phi)} d \theta
$$

and substituting (A-4) into (A-3), it yields

$$
\begin{aligned}
V\left(\rho, \theta_{s}\right) & =2 \pi \int_{0}^{1} T^{\prime}(r) \cdot J_{0}(2 \pi \rho \cdot r) \cdot r d r \\
& =300 \cdot 2 \pi \int_{0}^{0.15} J_{0}(2 \pi \rho \cdot r) \cdot r d r .
\end{aligned}
$$

Then, substituting $r^{\prime}=2 \pi \rho \cdot r$ into (A-6), it gives

$$
V\left(\rho, \theta_{s}\right)=\frac{300}{2 \pi \cdot \rho^{2}} \int_{0}^{0.15 \cdot 2 \pi \rho} J_{0}\left(r^{\prime}\right) r^{\prime} d r^{\prime} .
$$

Finally, considering the integral property between zeroorder and first-order Bessel functions $J_{0}(x)$ and $J_{1}(x)$

$$
\int_{0}^{z} x J_{0}(x) d x=z J_{1}(z),
$$


thus, the visibility function can be analytically computed by

$$
V\left(\rho, \theta_{s}\right)=\frac{45}{\rho} \cdot J_{1}\left(\frac{3 \pi}{10} \cdot \rho\right) .
$$

\section{APPENDIX B}

\section{EXPLANATION FOR THE STATISTICS OF AMPLitUde NOISE}

The amplitude noise presented in (14) can be rewritten in a simplified form as:

$$
n_{k l}^{A}=\ln \left|V_{c o r}+n_{k l}^{c}\right|-\ln \left|V_{c o r}\right| .
$$

It is well-known that when $V_{c o r} \rightarrow 0,\left|V_{c o r}+n_{k l}^{c}\right|$ can be approximated by a Rayleigh distribution. And its natural logarithm is referred to as the Log-Rayleigh distribution $Z=\ln \left|V_{c o r}+n_{k l}^{c}\right|$ defined in [17], which has the mean $m_{Z}$ and the variance $v_{Z}$ :

$$
\begin{gathered}
m_{Z}=\ln \sigma_{c}+\frac{\ln 2}{2}-\frac{\gamma}{2} \\
v_{Z}=\pi^{2} / 24
\end{gathered}
$$

where $\sigma_{c}$ is the standard deviation of the complex Gaussian noise $n_{k l}^{c}$, and $\gamma$ is the Euler constant.

1) The linear relationship between $S N R$ and mean value $(S N R<0 \quad d B):$

The mean value of $n_{k l}^{A}$ can be expressed as:

$$
\begin{aligned}
\mathrm{E}\left[n_{k l}^{A}\right] & =\mathrm{E}\left[\ln \left|V_{c o r}+n_{k l}^{c}\right|-\ln \left|V_{c o r}\right|\right] \\
& =\mathrm{E}\left[\ln \left|V_{c o r}+n_{k l}^{c}\right|\right]-\ln \left|V_{c o r}\right|
\end{aligned}
$$

using the definition $\mathrm{SNR}=\left|V_{\text {cor }}\right| / \sigma_{c}$ and the Rayleigh approximation in low-SNR cases, we derive that

$$
\begin{aligned}
\mathrm{E}\left[n_{k l}^{A}\right] & =\ln \sigma_{c}+\frac{\ln 2}{2}-\frac{\gamma}{2}-\ln \left[\sqrt{10^{\mathrm{SNR} / 10} * \sigma_{c}^{2}}\right] \\
& \approx 0.058-\frac{\ln (10)}{20} \cdot \mathrm{SNR}
\end{aligned}
$$

where the mean value of amplitude noise shows a linear relationship with respect to SNR.

2) The upper limit of standard deviation:

The standard deviation of $n_{k l}^{A}$ is equal to the standard deviation of $\ln \left|V_{\text {corr }}+n_{k l}^{c}\right|$, because the second term in the right-hand side of (14) can be considered as a constant. Using the Rayleigh approximation in low-SNR cases, the standard deviation of $n_{k l}^{A}$ can be approximated by the standard deviation of a Log-Rayleigh distribution:

$$
\sigma_{Z}=\sqrt{\pi^{2} / 24} \approx 0.6413
$$

The properties presented in (B-5) and (B-6) perfectly agrees with the Monte Carlo simulation results illustrated in Fig. 2.

\section{APPENDIX C \\ SuPPlEMENTARY Results FOR THE AMPLitude CALIBRATION}

A. Amplitude Calibration with Non-separable Amplitude Factors

When evaluating the simulation results shown in Fig. 14 and Fig. 15, the non-separable amplitude factors are included in the amplitude calibration residuals. Since the non-separable amplitude factors are set to have a standard deviation of $2 \%$, the calibration uncertainty is definitely larger than $2 \%$ in each case. To individually show the calibration uncertainty introduced by the estimated separable amplitude factors, the calibration uncertainty is evaluated when the non-separable amplitude factors are not included in the calibration residuals, as presented in Fig. 25.

The calibration uncertainty contributed by the misfit of the estimated separable amplitude factors is less than $1 \%$ in each case, and the performance of the different averaging methods is consistent with the results presented in Section V. Note that the amplitude calibration bias under this circumstance is not presented here, because the non-separable amplitude factors are set to have a mean value of 1 , and only negligible differences can be found.

The amplitude calibration performance can also be evaluated from the perspective of element's quantity. As we have found that averaging of the complex measurements in a complete array rotation shows the best calibration performance among all kinds of averaging methods, its performance versus the total number of elements is evaluated in Fig. 26. As a matter of fact, the increase of element's quantity does not make significant difference in the calibration bias, but the calibration uncertainty is reduced slightly.

\section{B. Amplitude Calibration without Non-separable Amplitude Factors}

As mentioned in Section. V, the amplitude calibration is affected by both thermal noise and the non-separable amplitude factors simultaneously. To show the influence of thermal noise individually, the case that non-separable amplitude factors are not considered during the amplitude calibration is simulated, which means that the non-separable amplitude factors are set to 1 when the complex cross-correlations are simulated.

Simulation results are shown in Fig. 27 and Fig. 28, respectively. The amplitude calibration bias also exists when there is not a non-separable amplitude factor during the calibration. Although we have designed the weighting function based on the noise behavior of the natural logarithm of the measured amplitude, the calibration residuals are still amplified considerably by the exponentiation involved in the de-linearization process.

\section{APPENDIX D}

\section{SupPlementary Results FOR THE Phase CALIBRATION}

The performance of the phase calibration is evaluated when a larger non-separable phase factor is considered, and the nonseparable phase factors are set by $\phi_{k l} \sim N\left(0, \sigma^{2}=1^{2}\right) \mathrm{deg}$. 


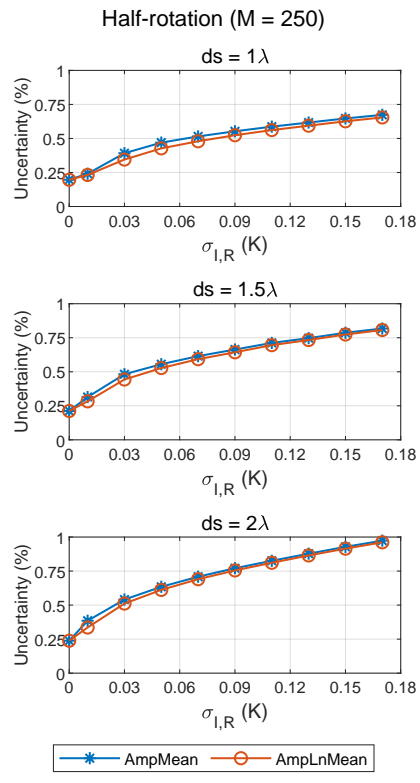

(a)
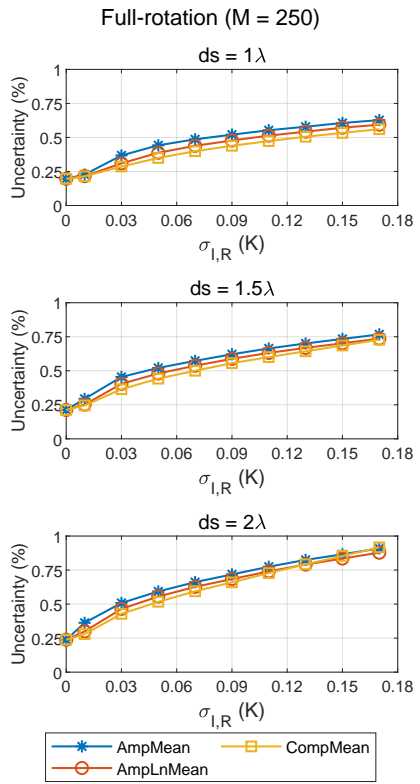

(b)

Fig. 25. Simulation results of the amplitude calibration uncertainty, the nonseparable amplitude factors are not included in the calibration residuals, (a) half-rotation; (b) full-rotation. The total number of elements $M$ is equal to 250 in each case (averaged of 300 Monte-Carlo runs).
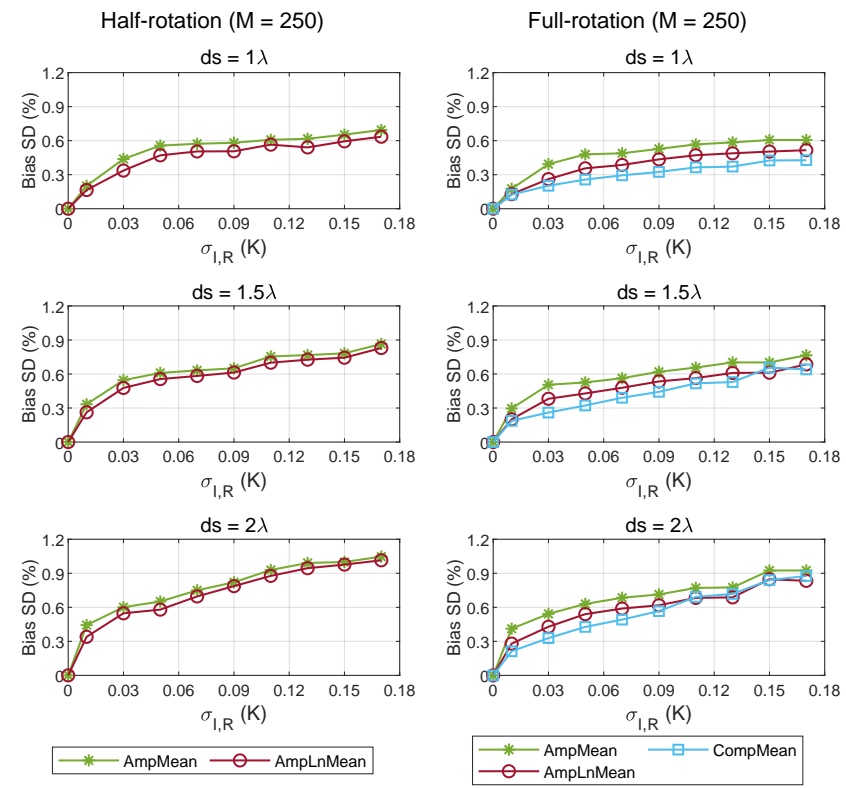

(a)

(b)

Fig. 27. Simulation results of the amplitude calibration bias, the nonseparable amplitude factors are not considered during the calibration, (a) half-rotation; (b) full-rotation. The total number of elements $M$ is equal to 250 in each case (averaged of 300 Monte-Carlo runs).

In order to compare the misfits of the estimated separable phase factors between two different configurations of the nonseparable phase factors, the non-separable phase factors are not included in the phase calibration residuals, and averaging of the complex measurements in a complete array rotation is used as the calibration scheme.

The simulation results are shown in Fig. 29, where the phase

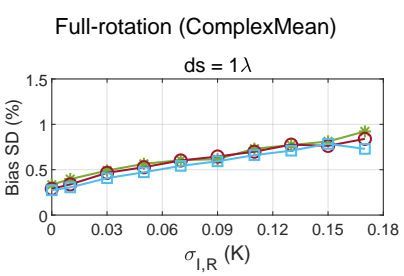

Full-rotation (ComplexMean)
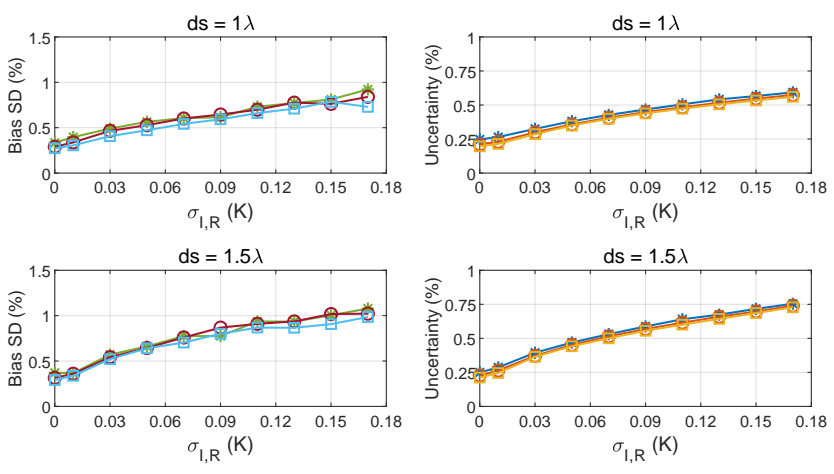

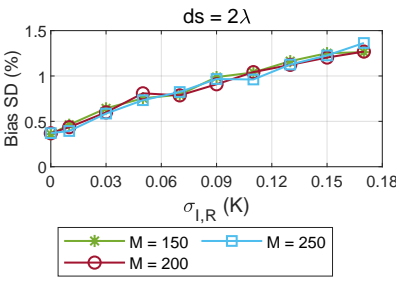

(a)

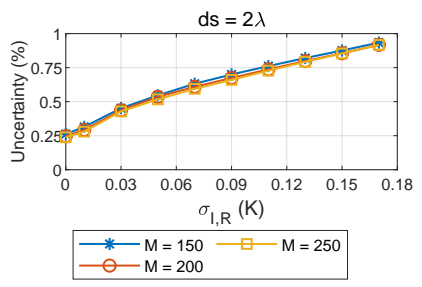

(b)
Fig. 26. Simulation results of (a) the amplitude calibration bias, (b) the amplitude uncertainty. The non-separable amplitude factors are not included in the calibration residuals (averaged of 300 Monte-Carlo runs).
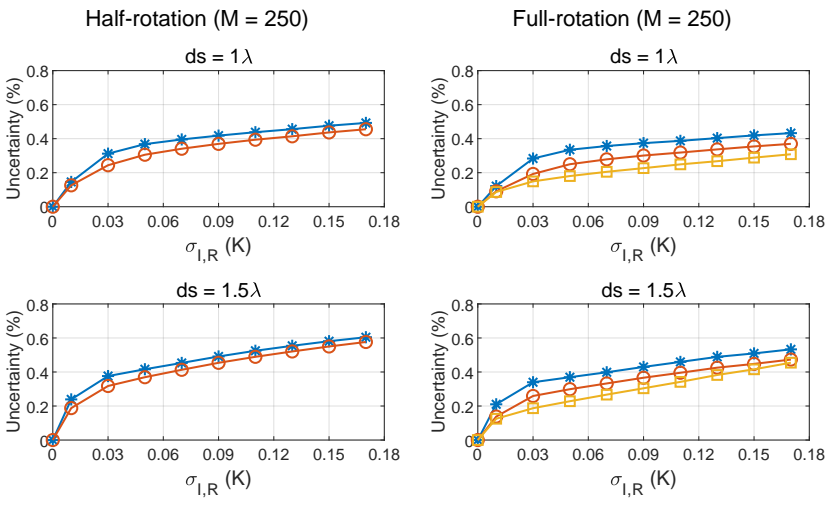

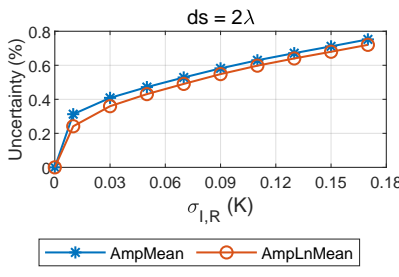

(a)

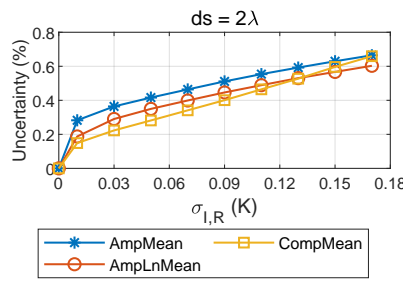

(b)
Fig. 28. Simulation results of the amplitude calibration uncertainty, the non-separable amplitude factors are not considered during the calibration, (a) half-rotation; (b) full-rotation. The total number of elements $M$ is equal to 250 in each case (averaged of 300 Monte-Carlo runs).

calibration shows an equivalent performance even the nonseparable phase factor is 6 times larger. Note that the data used to illustrate Fig. 29(a) are the same as the ones used in Fig. 9(b), the only difference is whether the non-separable phase factors are included in the phase residuals or not. 

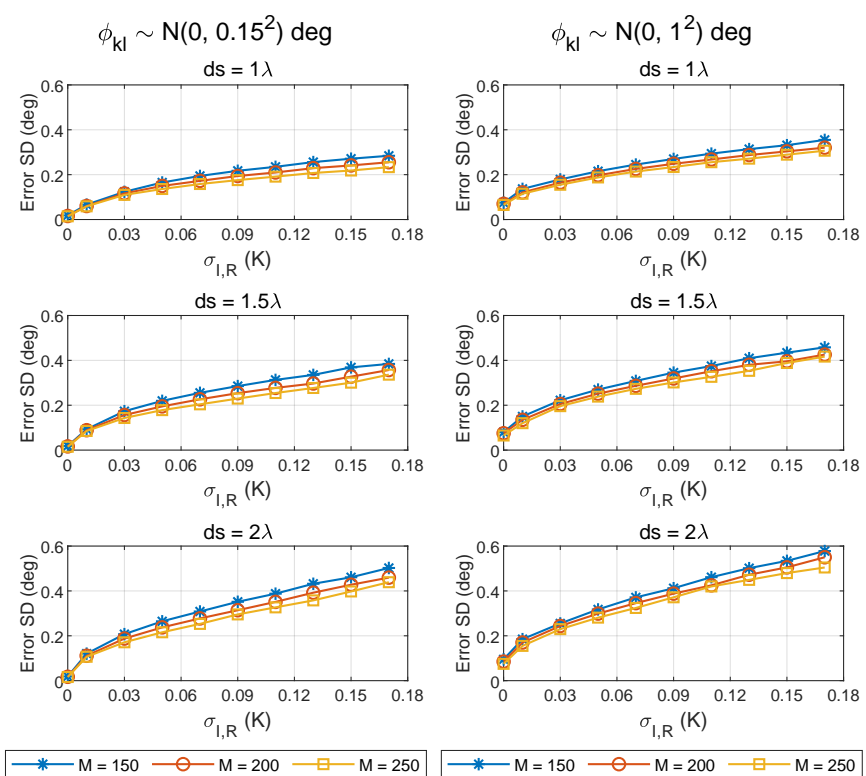

(a)

(b)

Fig. 29. Simulation results of phase calibration residuals, (a) $\phi_{k l} \sim$ $N\left(0,0.15^{2}\right) \mathrm{deg}$, and (b) $\phi_{k l} \sim N\left(0,1^{2}\right) \mathrm{deg}$. The non-separable phase factors are not included in the phase residuals (averaged of 300 Monte-Carlo runs).

\section{ACKNOWLEDGMENT}

The authors would like to thank the anonymous editor and reviewers for their constructive comments and suggestions. This collaboration was also supported by the Joint Ph.D. Training Program from the University of Chinese Academy of Sciences.

\section{REFERENCES}

[1] X. Guo, A. Camps, H. Park, H. Liu, C. Zhang, and J. Wu, "Phase and amplitude calibrations of rotating equispaced circular array for geostationary microwave interferometric radiometers-theory and methods," IEEE Transactions on Geoscience and Remote Sensing, pp. 1-15, 2021.

[2] J. Bará, A. Camps, F. Torres, and I. Corbella, "Angular resolution of twodimensional, hexagonally sampled interferometric radiometers," Radio Science, vol. 33, no. 5, pp. 1459-1473, Sept./Oct. 1998.

[3] I. Corbella, N. Duffo, M. Vall-llossera, A. Camps, and F. Torres, "The visibility function in interferometric aperture synthesis radiometry," IEEE Transactions on Geoscience and Remote Sensing, vol. 42, no. 8 , pp. 1677-1682, Aug. 2004.

[4] C. Zhang, H. Liu, J. Wu, S. Zhang, J. Yan, L. Niu, W. Sun, and H. Li, "Imaging analysis and first results of the geostationary interferometric microwave sounder demonstrator," IEEE Transactions on Geoscience and Remote Sensing, vol. 53, no. 1, pp. 207-218, Jan. 2015.

[5] F. Torres, A. Camps, J. Bará, and I. Corbella, "Impact of receiver errors on the radiometric resolution of large two-dimensional aperture synthesis radiometers," Radio Science, vol. 32, no. 2, pp. 629-641, Mar. 1997.

[6] X. Bosch-Lluis, I. Ramos-Perez, A. Camps, N. Rodriguez-Alvarez, E. Valencia, and H. Park, "A general analysis of the impact of digitization in microwave correlation radiometers," Sensors, vol. 11, no. 6, pp. 6066-6087, Jun. 2011.

[7] I. Corbella, F. Torres, N. Duffo, M. Martin-Neira, V. Gonzalez-Gambau, A. Camps, and M. Vall-1lossera, "On-ground characterization of the SMOS payload," IEEE Transactions on Geoscience and Remote Sensing, vol. 47, no. 9, pp. 3123-3133, Sept. 2009.

[8] C. S. Ruf, C. T. Swift, A. B. Tanner, and D. M. L. Vine, "Interferometric synthetic aperture microwave radiometry for the remote sensing of the earth," IEEE Transactions on Geoscience and Remote Sensing, vol. 26, no. 5, pp. 597-611, Sept. 1988.
[9] A. Camps, "Application of interferometric radiometry to earth observation," Ph.D. dissertation, Dept. de Teoria del Senyal i Comunicacions, Politècnica de Catalunya, Barcelona, Spain, 1996. [Online]. Available: "https://www.tdx.cat/handle/10803/6885"

[10] J. B. Hagen and D. T. Farley, "Digital-correlation techniques in radio science," Radio Science, vol. 8, no. 8-9, pp. 775-784, Aug./Sept. 1973.

[11] R. Butora and A. Camps, "Noise maps in aperture synthesis radiometric images due to cross-correlation of visibility noise," Radio Science, vol. 38, no. 4, pp. 6-1-6-8, Aug. 2003.

[12] A. Averbuch, R. R. Coifman, D. L. Donoho, M. Elad, and M. Israeli, "Fast and accurate polar fourier transform," Applied and Computational Harmonic Analysis, vol. 21, no. 2, pp. 145-167, 2006.

[13] C. Zhang, J. Wu, and W. Sun, "Applications of pseudo-polar FFT in synthetic aperture radiometer imaging," J PIERS online, vol. 3, no. 1 , pp. 25-30, 2007.

[14] A. R. Thompson, J. M. Moran, and G. W. Swenson, Interferometry and Synthesis in Radio Astronomy, ser. Interferometry and Synthesis in Radio Astronomy. Springer International Publishing, 2017.

[15] J. Bara, A. Camps, F. Torres, and I. Corbella, "The correlation of visibility noise and its impact on the radiometric resolution of an aperture synthesis radiometer," IEEE Transactions on Geoscience and Remote Sensing, vol. 38, no. 5, pp. 2423-2426, Sept. 2000.

[16] D. C. Montgomery, E. A. Peck, and G. G. Vining, Introduction to Linear Regression Analysis. Wiley \& Sons, 2012.

[17] P. Berens, "Circstat: A matlab toolbox for circular statistics," Journal of Statistical Software, Articles, vol. 31, no. 10, pp. 1-21, Sept. 2009.

[18] A. Camps, F. Torres, P. Lopez-Dekker, and S. J. Frasier, "Redundant space calibration of hexagonal and $\mathrm{Y}$-shaped beamforming radars and interferometric radiometers," International Journal of Remote Sensing, vol. 24, no. 24, pp. 5183-5196, 2003.

[19] I. Corbella, F. Torres, A. Camps, A. Colliander, M. Martin-Neira, S. Ribo, K. Rautiainen, N. Duffo, and M. Vall-llossera, "MIRAS end-toend calibration: application to SMOS L1 processor," IEEE Transactions on Geoscience and Remote Sensing, vol. 43, no. 5, pp. 1126-1134, Apr. 2005.

[20] A. Camps, H. Park, Y. Kang, J. Bandeiras, J. Barbosa, P. Vieira, A. Friaças, and S. D'Addio, "Microwave imaging radiometers by aperture synthesis performance simulator (Part 2): Instrument modeling, calibration, and image reconstruction algorithms," Journal of Imaging, vol. 2, no. 2, 2016

[21] Y. Zhang, H. Liu, J. Wu, J. He, and C. Zhang, "Statistical analysis for performance of detection and imaging of dynamic targets using the geostationary interferometric microwave sounder," IEEE Journal of Selected Topics in Applied Earth Observations and Remote Sensing, vol. 11, no. 1, pp. 3-11, Jan. 2018.

[22] H. Liu, L. Niu, C. Zhang, D. Han, H. Lu, X. Zhao, and J. Wu, "Preliminary results of gims-ii (geostationary interferometric microwave sounder-second generation) demonstrator," in 2017 IEEE International Geoscience and Remote Sensing Symposium (IGARSS), Dec. 2017, pp. $711-714$.

[23] A. K. S. E. Maghraby, H. Park, A. Camps, A. Grubišić, C. Colombo, and A. Tatnall, "Phase and baseline calibration for microwave interferometric radiometers using beacons," IEEE Transactions on Geoscience and Remote Sensing, pp. 1-12, Aug. 2020.

[24] H. Lu, Q. Li, R. Jin, K. Chen, Y. Li, L. Feng, H. Li, and Y. Li, "A hybrid calibration method for aperture synthesis radiometers," IEEE Geoscience and Remote Sensing Letters, vol. 13, no. 5, pp. 651-655, May 2016.

[25] R. Jin, Q. Li, K. Chen, G. Yi, H. Yang, D. Li, and J. Dong, "An onboard external calibration method for aperture synthesis radiometer by rotation," IEEE Geoscience and Remote Sensing Letters, vol. 9, no. 5, pp. 901-905, Sept. 2012.

[26] D. Han, H. Liu, J. Wu, Z. Cheng, L. Niu, and Y. Zhang, "Interelement phase calibration for the geostationary interferometric microwave sounder (GIMS)," IEEE Geoscience and Remote Sensing Letters, vol. 13, no. 9, pp. 1216-1220, Sept. 2016. 


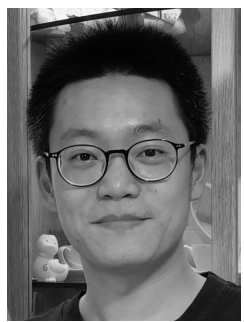

Xi Guo (Student Member, IEEE) was born in Taiyuan, China, in 1993. He received the B.S. degree in electronic engineering from Beijing Institute of Technology, Beijing, China, in 2015. He is currently pursuing the Ph.D. degree with the National Space Science Center, Chinese Academy of Sciences.

From 2019 to 2020, he was with the Remote Sensing Group, Polytechnic University of Catalonia, Barcelona, Spain, as a visiting Ph.D student. His research interests include passive remote sensing, with special emphasis on the calibration and imaging method of geostationary interferometric radiometers.

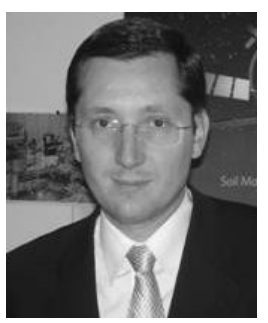

Adriano Camps (Fellow, IEEE) was born in Barcelona, Spain, in 1969. He received the B.S. and Ph.D. degrees in telecommunications engineering from the Universitat Politècnica de Catalunya (UPC), Barcelona, Spain, in 1992 and 1996, respectively.

In 1993, he joined the Electromagnetics and Photonics Engineering Group, Department of Signal Theory and Communications, UPC, as an Assistant Professor, an Associate Professor in 1997, and a Full Professor since 2007. In 1999, he was on sabbatical leave at the Microwave Remote Sensing Laboratory, University of Massachusetts Amherst, Amherst, MA, USA. Since 1993, he has been deeply involved with the European Space Agency Soil Moisture and Ocean Salinity (SMOS) Earth Explorer Mission, from the instrument and algorithmic points of view, performing field experiments, and since 2001 studying the use of Global Navigation Satellite Systems-Reflectometry (GNSS-R) techniques to perform the sea state correction needed to retrieve salinity from L-band radiometric observations. His research interests are focused on microwave remote sensing, with special emphasis on microwave radiometry by aperture synthesis techniques (MIRAS instrument onboard ESA's SMOS mission), remote sensing using signals of opportunity (GNSS-R), and nanosatellites as a tool to test innovative remote sensors. He has authored or coauthored over 227 articles in peer-reviewed journals, eight book chapters, and the book Emery and Camps, Introduction to Satellite Remote Sensing. Atmosphere, Ocean, Land and Cryosphere Applications (Elsevier, 2017, 860 pages), and more than 472 conference presentations, holds ten patents, and has advised $27 \mathrm{Ph} . \mathrm{D}$. thesis students (more than eight on-going), and more than 140 final projects and M.Eng. theses. According to Google Scholar/Scopus, his H-index is $52 / 40$.

Dr. Camps has received several awards, among which the European Young Investigator Award in 2004, the ICREA Academia research award in 2009 and 2015, and the Duran Farell award for Technology Transfer in 2000 and 2010.

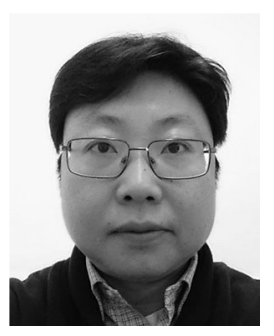

Hyuk Park (Senior Member, IEEE) was born in South Korea. He received the B.S. degree in mechanical engineering from the Korea Advanced Institute of Science and Technology (KAIST), Daejeon, Korea, in 2001 and the M.S. and Ph.D. degrees in information and mechatronics from Gwangju Institute of Science and Technology (GIST), Gwangju, Korea, in 2003 and 2009, respectively. In 2009, he joined the Remote Sensing Group, Polytechnic University of Catalonia (UPC), Barcelona, Spain, as a Post-Doctoral Researcher. In 2011, he was a grant holder of the National Research Foundation funded by the Korean Government. Since 2012, he has been working as a Research Associate with a Juan de la Cierva Grant funded by the Spanish Ministry of Economy and Competitiveness. He is the winner of the Chong-Hoon Cho Academic Award 2019 in Korea. He is working with the School of Telecommunications and Aerospace Engineering, Castelldefels (EETAC-UPC) as a Ramon y Cajal Fellow/a tenure-track Assistant Professor. He is also working with the Passive Remote Sensing Group, UPC, for satellite remote sensing for microwave radiometry and Global Navigation Satellite Systems reflectometry. His main research interests are in the area of remote sensing, particularly passive microwave remote sensing, including system design, modeling and simulation, image/data processing, and small satellite applications.

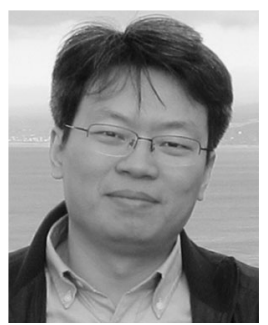

Hao Liu (Member, IEEE) was born in Jiujiang, China. He received the B.S. degree in information science and electronic engineering from Zhejiang University, Hangzhou, China, in 1999 and the M.S. degree in aircraft design and the Ph.D. degree in applied computer technology from the Graduate University of Chinese Academy of Sciences, Beijing, China, in 2002 and 2011, respectively.

In 2005, he was an International Trainee with the European Space Agency. He is a Research Professor with the CAS Key Laboratory of Microwave Remote Sensing, National Space Science Center, Chinese Academy of Sciences. His main research interests include microwave radiometry, particularly microwave interferometric radiometers (MIRs). His research interests include the prestudy and demonstration of MIR systems for future geostationary millimeter-wave atmospheric sounding mission and L-band ocean salinity mission.

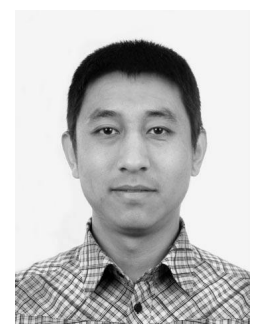

Cheng Zhang (Member, IEEE) received the B.E. degree in electronic engineering and the M.S. degree in electronic and information engineering from Hebei University of Technology, Tianjin, China, in 2001 and 2004, respectively, and the Ph.D. degree in applied computer technology from the Graduate University of Chinese Academy of Sciences (CAS), Beijing, China, in 2007.

Since 2007, he has been with the CAS Key Laboratory of Microwave Remote Sensing, National Space Science Center, CAS, where he was first an Assistant Professor and has been a Full Professor, since 2018. His research interests include antenna array design, numerical analysis, and image processing for microwave synthetic aperture interferometric radiometer.

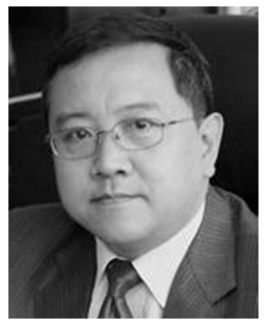

Ji Wu (Fellow, IEEE) was born in Beijing, China. He received the B.S. and M.S. degrees from the Beijing University of Posts and Telecommunications, Beijing, China, in 1985 and 1988, respectively, and the Ph.D. degree from the Technical University of Denmark, Copenhagen, Denmark, in 1993.

From 1985 to 1986 , he was with the European Space Research and Technology Centre, Noordwijk, The Netherlands, with a United Nations Research Fellowship, where he worked on contoured beam satellite antenna design. From 1986 to 1989 , he was with the Beijing University of Posts and Telecommunications, where he worked on antenna array optimization theory and design. From 19891994, he was with Technical University of Denmark, Lyngby, Denmark, as Ph.D. student and Postdoctoral Researcher. Since 1995, he has been with the CAS Key Laboratory of Microwave Remote Sensing, National Space Science Center, Chinese Academy of Sciences, Beijing. His current research interests include interferometric imaging technology for radiometer, laser space telecommunication and space science. 